\title{
Crystals of Lakshmibai-Seshadri paths and extremal weight modules over quantum hyperbolic Kac-Moody algebras of rank 2
}

\author{
Ryuta Hiasa \\ Graduate School of Pure and Applied Sciences, University of Tsukuba, \\ 1-1-1 Tennodai, Tsukuba, Ibaraki 305-8571, Japan \\ (e-mail: hiasa@math.tsukuba.ac.jp)
}

\begin{abstract}
Let $\mathfrak{g}$ be a hyperbolic Kac-Moody algebra of rank 2, and let $\lambda$ be an arbitrary integral weight. We denote by $\mathbb{B}(\lambda)$ the crystal of all Lakshmibai-Seshadri paths of shape $\lambda$. Let $V(\lambda)$ be the extremal weight module of extremal weight $\lambda$ generated by the (cyclic) extremal weight vector $v_{\lambda}$ of weight $\lambda$, and let $\mathcal{B}(\lambda)$ be the crystal basis of $V(\lambda)$ with $u_{\lambda} \in \mathcal{B}(\lambda)$ the element corresponding to $v_{\lambda}$. We prove that the connected component $\mathcal{B}_{0}(\lambda)$ of $\mathcal{B}(\lambda)$ containing $u_{\lambda}$ is isomorphic, as a crystal, to the connected component $\mathbb{B}_{0}(\lambda)$ of $\mathbb{B}(\lambda)$ containing the straight line $\pi_{\lambda}$. Furthermore, we prove that if $\lambda$ satisfies a special condition, then the crystal basis $\mathcal{B}(\lambda)$ is isomorphic, as a crystal, to the crystal $\mathbb{B}(\lambda)$. As an application of these results, we obtain an algorithm for computing the number of elements of weight $\mu$ in $\mathcal{B}\left(\Lambda_{1}-\Lambda_{2}\right)$, where $\Lambda_{1}, \Lambda_{2}$ are the fundamental weights, in the case that $\mathfrak{g}$ is symmetric.
\end{abstract}

\section{Introduction.}

Let $\mathfrak{g}$ be a symmetrizable Kac-Moody algebra over $\mathbb{C}$, and $U_{q}(\mathfrak{g})$ the quantized universal enveloping algebra over $\mathbb{C}(q)$ associated to $\mathfrak{g}$. We denote by $W$ the Weyl group of $\mathfrak{g}$. Let $P$ be an integral weight lattice of $\mathfrak{g}$, and $P^{+}\left(\right.$resp., $\left.-P^{+}\right)$the set of dominant (resp., antidominant) integral weights in $P$. Let $\mu \in P$ be an arbitrary integral weight. The extremal weight module $V(\mu)$ of extremal weight $\mu$ is the integrable $U_{q}(\mathfrak{g})$-module generated by a single element $v_{\mu}$ with the defining relation that $v_{\mu}$ is an extremal weight vector of weight $\mu$ in the sense of [5]. This module was introduced by Kashiwara [5] as a natural generalization of integrable highest (or lowest) weight modules; in fact, if $\mu \in P^{+}$(resp., $\mu \in-P^{+}$), then the extremal weight module of extremal weight $\mu$ is isomorphic, as a $U_{q}(\mathfrak{g})$-module, to the integrable highest (resp., lowest) weight module of highest (resp., lowest) weight $\mu$. Also, he proved in [5, Proposition 8.2.2] that $V(\mu)$ has 
a crystal basis $\mathcal{B}(\mu)$ for all $\mu \in P$; let $u_{\mu}$ denote the element of $\mathcal{B}(\mu)$ corresponding to $v_{\mu} \in V(\mu)$. We know from [5] that $V(\mu) \cong V(w \mu)$ as $U_{q}(\mathfrak{g})$-modules, and $\mathcal{B}(\mu) \cong \mathcal{B}(w \mu)$ as crystals for all $\mu \in P$ and $w \in W$. Hence we are interested in the case that

$$
W \mu \cap\left(P^{+} \cup-P^{+}\right)=\emptyset .
$$

If $\mathfrak{g}$ is of finite type, then $W \mu \cap P^{+} \neq \emptyset$ for any $\mu \in P$. Assume that $\mathfrak{g}$ is of affine type. Then, $W \mu \cap\left(P^{+} \cup-P^{+}\right)=\emptyset$ if and only if $(\mu \neq 0$, and $) \mu$ is of level zero. Naito and Sagaki proved in [9] and [10] that if $\mu$ is a positive integer multiple of a level-zero fundamental weight, then the crystal basis $\mathcal{B}(\mu)$ of the extremal weight module $V(\mu)$ is isomorphic, as a crystal, to the crystal $\mathbb{B}(\mu)$ of Lakshmibai-Seshadri (LS for short) paths, which was introduced by Littelmann in [12] and [13]; see $\$ 2.4$ for the details. After that, Ishii, Naito, and Sagaki [3] introduced the notion of semi-infinite LS paths of shape $\mu$ for a level-zero dominant integral weight $\mu$, and proved that the crystal basis $\mathcal{B}(\mu)$ of the extremal weight module $V(\mu)$ is isomorphic, as a crystal, to the crystal $\mathbb{B}^{\frac{\infty}{2}}(\mu)$ of semi-infinite LS paths of shape $\mu$. Now, we assume that $\mathfrak{g}$ is the hyperbolic Kac-Moody algebra associated to the generalized Cartan matrix

$$
A=\left(\begin{array}{cc}
2 & -a_{1} \\
-a_{2} & 2
\end{array}\right), \quad \text { where } a_{1}, a_{2} \in \mathbb{Z}_{\geq 2} \text { with } a_{1} a_{2}>4 .
$$

$\mathrm{Yu}$ [15] proved that $\Lambda_{1}-\Lambda_{2} \in P$ satisfies condition (1.1), where $\Lambda_{1}, \Lambda_{2}$ are the fundamental weights, and that (the crystal graph of) $\mathbb{B}\left(\Lambda_{1}-\Lambda_{2}\right)$ is connected. Then, Sagaki and Yu [14] proved that $\mathbb{B}\left(\Lambda_{1}-\Lambda_{2}\right)$ is isomorphic, as a crystal, to the crystal basis $\mathcal{B}\left(\Lambda_{1}-\Lambda_{2}\right)$ of the extremal weight module $V\left(\Lambda_{1}-\Lambda_{2}\right)$ of extremal weight $\Lambda_{1}-\Lambda_{2}$. In [1, the author obtained the following necessary and sufficient condition for an integral weight to satisfy condition (1.1): Let $\mathbb{O}:=\{W \mu \mid \mu \in P\}$ be the set of $W$-orbits in $P$. A $W$-orbit $O \in \mathbb{O}$ satisfies condition (1.1), that is, $O \cap\left(P^{+} \cup-P^{+}\right)=\emptyset$ if and only if $O$ contains an integral weight of the form either (ii) or (iii):

(i) $k_{1} \Lambda_{1}-k_{2} \Lambda_{2}$ for some $k_{1}, k_{2} \in \mathbb{Z}_{>0}$ such that $k_{2} \leq k_{1}<\left(a_{1}-1\right) k_{2}$;

(ii) $k_{1} \Lambda_{1}-k_{2} \Lambda_{2}$ for some $k_{1}, k_{2} \in \mathbb{Z}_{>0}$ such that $k_{1}<k_{2} \leq\left(a_{2}-1\right) k_{1}$.

Also, he proved that for $\lambda=k_{1} \Lambda_{1}-k_{2} \Lambda_{2} \in P$ of the form either (ii) or (ii) above, the crystal graph $\mathbb{B}(\lambda)$ is connected if and only if $k_{1}=1$ or $k_{2}=1$.

In this paper, we study the relationship between the crystal $\mathbb{B}(\lambda)$ of LS paths of shape $\lambda$ and the crystal basis $\mathcal{B}(\lambda)$ of extremal weight module $V(\lambda)$ of extremal weight $\lambda$ in the case that $\lambda=k_{1} \Lambda_{1}-k_{2} \Lambda_{2}$ is of the form either (ii) or (iii) above. We prove the following theorems.

Theorem 1.1 (= Theorem [3.2). Let $\mathbb{B}_{0}(\lambda)$ (resp., $\mathcal{B}_{0}(\lambda)$ ) be the connected component of $\mathbb{B}(\lambda)$ (resp., $\mathcal{B}(\lambda)$ ) containing $\pi_{\lambda}:=(\lambda ; 0,1)$ (resp., $\left.u_{\lambda}\right)$. There exists an isomorphism $\mathbb{B}_{0}(\lambda) \rightarrow \mathcal{B}_{0}(\lambda)$ of crystals that sends $\pi_{\lambda}$ to $u_{\lambda}$. 
Theorem 1.2 (= Theorem 3.3). If $k_{1}=1$ or $k_{2}=1$, that is, $\lambda \in P$ is of the form either $k_{1} \Lambda_{1}-\Lambda_{2}$ with $1 \leq k_{1}<a_{1}-1$ or $\Lambda_{1}-k_{2} \Lambda_{2}$ with $1<k_{2} \leq a_{2}-1$, then $\mathcal{B}(\lambda)$ is connected.

Let $\lambda$ be as in Theorem [1.2. By [1, Theorem 4.1] (resp., Theorem 1.2), we have $\mathbb{B}(\lambda)=\mathbb{B}_{0}(\lambda)$ (resp., $\mathcal{B}(\lambda)=\mathcal{B}_{0}(\lambda)$ ). Therefore, by Theorem 1.1, we obtain the following corollary.

Corollary 1.3 (= Corollary 3.4). If $k_{1}=1$ or $k_{2}=1$, then there exists an isomorphism $\mathbb{B}(\lambda) \rightarrow \mathcal{B}(\lambda)$ of crystals that sends $\pi_{\lambda}$ to $u_{\lambda}$.

As an application of these results, we give an algorithm for computing the number of elements of weight $\mu \in P$ in the crystal $\mathcal{B}\left(\Lambda_{1}-\Lambda_{2}\right)$, which is equal to the dimension of the weight space of weight $\mu$ in $V\left(\Lambda_{1}-\Lambda_{2}\right)$, in the case that $A$ is symmetric, that is $a_{1}=a_{2}$.

This paper is organized as follows. In Section 2, we fix our notation, and recall some basic facts about extremal weight modules and their crystal bases. Also, we recall the definition of LS paths and the polyhedral realizations of $\mathcal{B}( \pm \infty)$. In Section 3, we state our main theorems. In Section 4, we recall some properties of LS paths and the polyhedral realizations in the rank 2 case. Then we prove Theorems 1.1 and 1.2 in Subsections 5.1 and 5.2, respectively. In Section 6, we give an algorithm for computing the number of elements of weight $\mu \in P$ in the crystal $\mathcal{B}\left(\Lambda_{1}-\Lambda_{2}\right)$.

\section{Review.}

\subsection{Kac-Moody algebras.}

Let $A$ be a generalized Cartan matrix and $\mathfrak{g}=\mathfrak{g}(A)$ the Kac-Moody algebra associated to $A$ over $\mathbb{C}$. We denote by $\mathfrak{h}$ the Cartan subalgebra of $\mathfrak{g},\left\{\alpha_{i}\right\}_{i \in I} \subset \mathfrak{h}^{*}$ the set of simple roots, and $\left\{\alpha_{i}^{\vee}\right\}_{i \in I} \subset \mathfrak{h}$ the set of simple coroots, where $I$ is the index set. Let $s_{i}$ be the simple reflection with respect to $\alpha_{i}$ for $i \in I$, and let $W=\left\langle s_{i} \mid i \in I\right\rangle$ be the Weyl group of $\mathfrak{g}$. Let $\Delta_{\mathrm{re}}^{+}$denote the set of positive real roots. For a positive real root $\beta \in \Delta_{\mathrm{re}}^{+}$, we denote by $\beta^{\vee}$ the dual root of $\beta$, and by $s_{\beta} \in W$ the reflection with respect to $\beta$. Let $\left\{\Lambda_{i}\right\}_{i \in I} \subset \mathfrak{h}^{*}$ be the fundamental weights for $\mathfrak{g}$, i.e., $\left\langle\Lambda_{i}, \alpha_{j}^{\vee}\right\rangle=\delta_{i, j}$ for $i, j \in I$, where $\langle\cdot, \cdot\rangle: \mathfrak{h}^{*} \times \mathfrak{h} \rightarrow \mathbb{C}$ is the canonical pairing of $\mathfrak{h}^{*}$ and $\mathfrak{h}$. We take an integral weight lattice $P$ containing $\alpha_{i}$ and $\Lambda_{i}$ for all $i \in I$. We denote by $P^{+}$(resp., $-P^{+}$) the set of dominant (resp., antidominant) integral weights.

Let $U_{q}(\mathfrak{g})$ be the quantized universal enveloping algebra over $\mathbb{C}(q)$ associated to $\mathfrak{g}$, and let $U_{q}^{+}(\mathfrak{g})$ (resp., $\left.U_{q}^{-}(\mathfrak{g})\right)$ be the positive (resp., negative) part of $U_{q}(\mathfrak{g})$, that is, $\mathbb{C}(q)$ subalgebra generated by the Chevalley generators $E_{i}$ (resp., $F_{i}$ ) of $U_{q}(\mathfrak{g})$ corresponding to the positive (resp., negative) simple root $\alpha_{i}$ (resp., $-\alpha_{i}$ ) for $i \in I$. 


\subsection{Crystal bases and crystals.}

For details on crystal bases and crystals, we refer the reader to [7] and [2]. Let $\mathcal{B}(\infty)$ (resp., $\mathcal{B}(-\infty)$ ) be the crystal basis of $U_{q}^{-}(\mathfrak{g})$ (resp., $U_{q}^{+}(\mathfrak{g})$ ), and let $u_{\infty} \in \mathcal{B}(\infty)$ (resp., $u_{-\infty} \in \mathcal{B}(-\infty)$ ) be the element corresponding to $1 \in U_{q}^{-}(\mathfrak{g})$ (resp., $1 \in U_{q}^{+}(\mathfrak{g})$ ). Denote by $*: \mathcal{B}( \pm \infty) \rightarrow \mathcal{B}( \pm \infty)$ the $*$-operation on $\mathcal{B}( \pm \infty)$; see [6, Theorem 2.1.1] and [7, §8.3]. For $\mu \in P$, let $\mathcal{T}_{\mu}=\left\{t_{\mu}\right\}$ be the crystal consisting of a single element $t_{\mu}$ such that

$$
\operatorname{wt}\left(t_{\mu}\right)=\mu, \quad \tilde{e}_{i} t_{\mu}=\tilde{f}_{i} t_{\mu}=\mathbf{0}, \quad \varepsilon_{i}\left(t_{\mu}\right)=\varphi_{i}\left(t_{\mu}\right)=-\infty \text { for } i \in I,
$$

where $\mathbf{0}$ is an extra element not contained in any crystal.

Let $B$ be a normal crystal in the sense of [5, §1.5]. We know from [5, §7] (see also [7, Theorem 11.1]) that $B$ has an action of the Weyl group $W$ as follows. For $i \in I$ and $b \in B$, we set

$$
S_{i} b:= \begin{cases}\tilde{f}_{i}^{\left\langle\operatorname{wt}(b), \alpha_{i}^{\vee}\right\rangle} b & \text { if }\left\langle\operatorname{wt}(b), \alpha_{i}^{\vee}\right\rangle \geq 0, \\ \tilde{e}_{i}^{-\left\langle\operatorname{wt}(b), \alpha_{i}^{\vee}\right\rangle} b & \text { if }\left\langle\operatorname{wt}(b), \alpha_{i}^{\vee}\right\rangle \leq 0 .\end{cases}
$$

Then, for $w \in W$, we set $S_{w}:=S_{i_{1}} \cdots S_{i_{k}}$ if $w=s_{i_{1}} \cdots s_{i_{k}}$. Notice that $\operatorname{wt}\left(S_{w} b\right)=w \operatorname{wt}(b)$ for $w \in W$ and $b \in B$.

Definition 2.1. An element of a normal crystal $B$ is said to be extremal if for each $w \in W$ and $i \in I$,

$$
\begin{aligned}
& \tilde{e}_{i}\left(S_{w} b\right)=\mathbf{0} \text { if }\left\langle\operatorname{wt}\left(S_{w} b\right), \alpha_{i}^{\vee}\right\rangle \geq 0 \\
& \tilde{f}_{i}\left(S_{w} b\right)=\mathbf{0} \text { if }\left\langle\operatorname{wt}\left(S_{w} b\right), \alpha_{i}^{\vee}\right\rangle \leq 0
\end{aligned}
$$

\subsection{Crystal bases of extremal weight modules.}

Let $\mu \in P$ be an arbitrary integral weight. The extremal weight module $V(\mu)$ of extremal weight $\mu$ is, by definition, the integrable $U_{q}(\mathfrak{g})$-module generated by a single element $v_{\mu}$ with the defining relation that $v_{\mu}$ is an extremal weight vector of weight $\mu$ in the sense of [5, Definition 8.1.1]. We know from [5, Proposition 8.2.2] that $V(\mu)$ has a crystal basis $\mathcal{B}(\mu)$. Let $u_{\mu}$ denote the element of $\mathcal{B}(\mu)$ corresponding to $v_{\mu}$.

Remark 2.2. We see from [5, Proposition 8.2 .2 (iv) and (v)] that $V(\mu) \cong V(w \mu)$ as $U_{q}(\mathfrak{g})$-modules, and $\mathcal{B}(\mu) \cong \mathcal{B}(w \mu)$ as crystals for all $\mu \in P$ and $w \in W$. Also, we know from the comment at the end of [5, §8.2] that if $\mu \in P^{+}$(resp., $\mu \in-P^{+}$), then $V(\mu)$ is isomorphic, as a $U_{q}(\mathfrak{g})$-module, to the integrable highest (resp., lowest) weight module of highest (resp., lowest) weight $\mu$, and $\mathcal{B}(\mu)$ is isomorphic, as a crystal, to its crystal basis. So, we focus on those $\mu \in P$ satisfying the condition that

$$
W \mu \cap\left(P^{+} \cup-P^{+}\right)=\emptyset
$$


The crystal basis $\mathcal{B}(\mu)$ of $V(\mu)$ can be realized (as a crystal) as follows. We set

$$
\mathcal{B}:=\bigsqcup_{\mu \in P} \mathcal{B}(\infty) \otimes \mathcal{T}_{\mu} \otimes \mathcal{B}(-\infty)
$$

in fact, $\mathcal{B}$ is isomorphic, as a crystal, to the crystal basis $\mathcal{B}\left(\tilde{U}_{q}(\mathfrak{g})\right)$ of the modified quantized universal enveloping algebra $\tilde{U}_{q}(\mathfrak{g})$ associated to $\mathfrak{g}$ (see [5, Theorem 3.1.1]). Denote by $*: \mathcal{B} \rightarrow \mathcal{B}$ the $*$-operation on $\mathcal{B}$ (see [5, Theorem 4.3.2]); we know from [5, Corollary 4.3.3] that for $b_{1} \in \mathcal{B}(\infty), b_{2} \in \mathcal{B}(-\infty)$, and $\mu \in P$,

$$
\left(b_{1} \otimes t_{\mu} \otimes b_{2}\right)^{*}=b_{1}^{*} \otimes t_{-\mu-\mathrm{wt}\left(b_{1}\right)-\mathrm{wt}\left(b_{2}\right)} \otimes b_{2}^{*}
$$

Remark 2.3. The weight of $\left(b_{1} \otimes t_{\mu} \otimes b_{2}\right)^{*}$ is equal to $-\mu$ for all $b_{1} \in \mathcal{B}(\infty)$ and $b_{2} \in$ $\mathcal{B}(-\infty)$ since $\operatorname{wt}\left(b_{1}^{*}\right)=\mathrm{wt}\left(b_{1}\right)$ and $\mathrm{wt}\left(b_{2}^{*}\right)=\mathrm{wt}\left(b_{2}\right)$.

Because $\mathcal{B}$ is a normal crystal by [5, $\S 2.1$ and Theorem 3.1.1], $\mathcal{B}$ has the action of the Weyl group $W$ (see \$2.2). We know the following proposition from [5, Proposition 8.2.2 (and Theorem 3.1.1)].

Theorem 2.4. For $\mu \in P$, the subset

$$
\left\{b \in \mathcal{B}(\infty) \otimes \mathcal{T}_{\mu} \otimes \mathcal{B}(-\infty) \mid b^{*} \text { is extremal }\right\}
$$

is a subcrystal of $\mathcal{B}(\infty) \otimes \mathcal{T}_{\mu} \otimes \mathcal{B}(-\infty)$, and is isomorphic, as a crystal, to the crystal basis $\mathcal{B}(\mu)$ of the extremal weight module $V(\mu)$ of extremal weight $\mu$. In particular, $u_{\infty} \otimes t_{\mu} \otimes$ $u_{-\infty} \in \mathcal{B}(\infty) \otimes \mathcal{T}_{\mu} \otimes \mathcal{B}(-\infty)$ is contained in the set above, and corresponds to $u_{\mu} \in \mathcal{B}(\mu)$ under the isomorphism.

\subsection{Lakshmibai-Seshadri paths.}

We recall Lakshmibai-Seshadri paths from [13, §2, §4]. In this subsection, we fix an integral weight $\mu \in P$.

Definition 2.5. For $\nu, \nu^{\prime} \in W \mu$, we write $\nu \geq \nu^{\prime}$ if there exist a sequence $\nu=\nu_{0}, \nu_{1}, \ldots$, $\nu_{u}=\nu^{\prime}$ of elements in $W \mu$ and a sequence $\beta_{1}, \beta_{2}, \ldots, \beta_{u}$ of positive real roots such that $\nu_{k}=s_{\beta_{k}}\left(\nu_{k-1}\right)$ and $\left\langle\nu_{k-1}, \beta_{k}^{\vee}\right\rangle<0$ for each $k=1,2, \ldots, u$. If $\nu \geq \nu^{\prime}$, then we define $\operatorname{dist}\left(\nu, \nu^{\prime}\right)$ to be the maximal length $u$ of all possible such sequences $\nu=\nu_{0}, \nu_{1}, \ldots, \nu_{u}=\nu^{\prime}$.

Remark 2.6. For $\nu, \nu^{\prime} \in W \mu$ such that $\nu>\nu^{\prime}$ and $\operatorname{dist}\left(\nu, \nu^{\prime}\right)=1$, there exists a unique positive real root $\beta \in \Delta_{\text {re }}^{+}$such that $\nu^{\prime}=s_{\beta}(\nu)$.

The Hasse diagram of $W \mu$ is, by definition, the $\Delta_{\mathrm{re}}^{+}$-labeled, directed graph with vertex set $W \mu$, and edges of the following form: $\nu \stackrel{\beta}{\leftarrow} \nu^{\prime}$ for $\nu, \nu^{\prime} \in W \mu$ and $\beta \in \Delta_{\text {re }}^{+}$such that $\nu>\nu^{\prime}$ with $\operatorname{dist}\left(\nu, \nu^{\prime}\right)=1$ and $\nu^{\prime}=s_{\beta}(\nu)$. 
Definition 2.7. Let $\nu, \nu^{\prime} \in W \mu$ with $\nu>\nu^{\prime}$, and let $0<\sigma<1$ be a rational number. A $\sigma$-chain for $\left(\nu, \nu^{\prime}\right)$ is a sequence $\nu=\nu_{0}, \ldots, \nu_{u}=\nu^{\prime}$ of elements of $W \mu$ such that $\operatorname{dist}\left(\nu_{k-1}, \nu_{k}\right)=1$ and $\sigma\left\langle\nu_{k-1}, \beta_{k}^{\vee}\right\rangle \in \mathbb{Z}_{<0}$ for all $k=1,2, \ldots, u$, where $\beta_{k}$ is the unique positive real root satisfying $\nu_{k}=s_{\beta_{k}}\left(\nu_{k-1}\right)$.

Definition 2.8. Let $\nu_{1}>\cdots>\nu_{u}$ be a finite sequence of elements in $W \mu$, and let $0=\sigma_{0}<\cdots<\sigma_{u}=1$ be a finite sequence of rational numbers. The pair $\pi=$ $\left(\nu_{1}, \ldots, \nu_{u} ; \sigma_{0}, \ldots, \sigma_{u}\right)$ is called a Lakshmibai-Seshadri (LS for short) path of shape $\nu$ if there exists a $\sigma_{k}$-chain for $\left(\nu_{k}, \nu_{k+1}\right)$ for each $k=1, \ldots, u-1$. We denote by $\mathbb{B}(\mu)$ the set of LS paths of shape $\mu$.

Let $[0,1]:=\{t \in \mathbb{R} \mid 0 \leq t \leq 1\}$. We identify $\pi=\left(\nu_{1}, \ldots, \nu_{u} ; \sigma_{0}, \ldots, \sigma_{u}\right) \in \mathbb{B}(\mu)$ with the following piecewise-linear continuous map $\pi:[0,1] \rightarrow \mathbb{R} \otimes_{\mathbb{Z}} P$ :

$$
\pi(t)=\sum_{k=1}^{j-1}\left(\sigma_{k}-\sigma_{k-1}\right) \nu_{k}+\left(t-\sigma_{j-1}\right) \nu_{j} \quad \text { for } \sigma_{j-1} \leq t \leq \sigma_{j}, 1 \leq j \leq u .
$$

We endow $\mathbb{B}(\mu)$ with a crystal structure as follows. First, we define $\operatorname{wt}(\pi):=\pi(1)$ for $\pi \in \mathbb{B}(\mu)$; we know from [13, Lemma 4.5 (a)] that $\pi(1) \in P$. Next, for $\pi \in \mathbb{B}(\mu)$ and $i \in I$,

$$
\begin{aligned}
H_{i}^{\pi}(t) & :=\left\langle\pi(t), \alpha_{i}^{\vee}\right\rangle \quad \text { for } 0 \leq t \leq 1, \\
m_{i}^{\pi} & :=\min \left\{H_{i}^{\pi}(t) \mid 0 \leq t \leq 1\right\} .
\end{aligned}
$$

From [13, Lemma 4.5 (d)], we know that

$$
\text { all local minimum values of } H_{i}^{\pi}(t) \text { are integers; }
$$

in particular, $m_{i}^{\pi} \in \mathbb{Z}_{\leq 0}$ and $H_{i}^{\pi}(1)-m_{i}^{\pi} \in \mathbb{Z}_{\geq 0}$. We define $\tilde{e}_{i} \pi$ as follows. If $m_{i}^{\pi}=0$, then we set $\tilde{e}_{i} \pi:=\mathbf{0}$. If $m_{i}^{\pi} \leq-1$, then we set

$$
\begin{aligned}
& t_{1}:=\min \left\{t \in[0,1] \mid H_{i}^{\pi}(t)=m_{i}^{\pi}\right\}, \\
& t_{0}:=\max \left\{t \in\left[0, t_{1}\right] \mid H_{i}^{\pi}(t)=m_{i}^{\pi}+1\right\} ;
\end{aligned}
$$

we see by (2.3) that

$$
H_{i}^{\pi}(t) \text { is strictly decreasing on }\left[t_{0}, t_{1}\right] \text {. }
$$

We define

$$
\left(\tilde{e}_{i} \pi\right)(t):= \begin{cases}\pi(t) & \text { if } 0 \leq t \leq t_{0} \\ s_{i}\left(\pi(t)-\pi\left(t_{0}\right)\right)+\pi\left(t_{0}\right) & \text { if } t_{0} \leq t \leq t_{1} \\ \pi(t)+\alpha_{i} & \text { if } t_{1} \leq t \leq 1\end{cases}
$$


we know from [13, $\S 4]$ that $\tilde{e}_{i} \pi \in \mathbb{B}(\mu)$. Similarly, we define $\tilde{f}_{i} \pi$ as follows. If $H_{i}^{\pi}(1)-m_{i}^{\pi}=$ 0 , then we set $\tilde{f}_{i} \pi:=\mathbf{0}$. If $H_{i}^{\pi}(1)-m_{i}^{\pi} \geq 1$, then we set

$$
\begin{aligned}
& t_{0}:=\max \left\{t \in[0,1] \mid H_{i}^{\pi}(t)=m_{i}^{\pi}\right\}, \\
& t_{1}:=\min \left\{t \in\left[t_{0}, 1\right] \mid H_{i}^{\pi}(t)=m_{i}^{\pi}+1\right\}
\end{aligned}
$$

we see by (2.3) that $H_{i}^{\pi}(t)$ is strictly increasing on $\left[t_{0}, t_{1}\right]$. We define

$$
\left(\tilde{f}_{i} \pi\right)(t):= \begin{cases}\pi(t) & \text { if } 0 \leq t \leq t_{0} \\ s_{i}\left(\pi(t)-\pi\left(t_{0}\right)\right)+\pi\left(t_{0}\right) & \text { if } t_{0} \leq t \leq t_{1} \\ \pi(t)-\alpha_{i} & \text { if } t_{1} \leq t \leq 1\end{cases}
$$

we know from [13, $\S 4]$ that $f_{i} \pi \in \mathbb{B}(\mu)$. We set $\tilde{e}_{i} \mathbf{0}=\tilde{f}_{i} \mathbf{0}:=\mathbf{0}$ for $i \in I$. Finally, for $\pi \in \mathbb{B}(\mu)$ and $i \in I$, we set

$$
\varepsilon_{i}(\pi):=\max \left\{k \in \mathbb{Z}_{\geq 0} \mid \tilde{e}_{i}^{k} \pi \neq \mathbf{0}\right\}, \quad \varphi_{i}(\pi):=\max \left\{k \in \mathbb{Z}_{\geq 0} \mid \tilde{f}_{i}^{k} \pi \neq \mathbf{0}\right\} .
$$

We know from [13, Lemma 2.1 (c)] that

$$
\varepsilon_{i}(\pi)=-m_{i}^{\pi}, \quad \varphi_{i}(\pi)=H_{i}^{\pi}(1)-m_{i}^{\pi} .
$$

Theorem $2.9([13, \S 2, \S 4])$. The set $\mathbb{B}(\mu)$, together with the maps wt $: \mathbb{B}(\mu) \rightarrow P$, $\tilde{e}_{i}, \tilde{f}_{i}: \mathbb{B}(\mu) \rightarrow \mathbb{B}(\mu) \cup\{\mathbf{0}\}, i \in I$, and $\varepsilon_{i}, \varphi_{i}: \mathbb{B}(\mu) \rightarrow \mathbb{Z}_{\geq 0}, i \in I$, is a crystal.

\subsection{Polyhedral realization of $\mathcal{B}( \pm \infty)$ and $\mathcal{B}(\infty) \otimes \mathcal{T}_{\mu} \otimes \mathcal{B}(-\infty)$.}

Let us recall the polyhedral realization of $\mathcal{B}(\infty)$ and $\mathcal{B}(-\infty)$ from [11]. We fix an infinite sequence $\iota^{+}=\left(\ldots, i_{k}, \ldots, i_{2}, i_{1}\right)$ of elements of $I$ such that $i_{k} \neq i_{k+1}$ for $k \in \mathbb{Z}_{\geq 1}$, and $\#\left\{k \in \mathbb{Z}_{\geq 1} \mid i_{k}=i\right\}=\infty$ for each $i \in I$. Similarly, we fix an infinite sequence $\iota^{-}=\left(i_{0}, i_{-1}, \ldots, i_{k}, \ldots\right)$ of elements of $I$ such that $i_{k} \neq i_{k-1}$ for $k \in \mathbb{Z}_{\leq 0}$, and $\#\left\{k \in \mathbb{Z}_{\leq 0} \mid\right.$ $\left.i_{k}=i\right\}=\infty$ for each $i \in I$. We set

$$
\begin{aligned}
& \mathbb{Z}_{\geq 0}^{+\infty}:=\left\{\left(\ldots, y_{k}, \ldots, y_{2}, y_{1}\right) \mid y_{k} \in \mathbb{Z}_{\geq 0} \text { and } y_{k}=0 \text { for } k \gg 0\right\}, \\
& \mathbb{Z}_{\leq 0}^{-\infty}:=\left\{\left(y_{0}, y_{-1}, \ldots, y_{k}, \ldots\right) \mid y_{k} \in \mathbb{Z}_{\leq 0} \text { and } y_{k}=0 \text { for } k \ll 0\right\} .
\end{aligned}
$$

We endow $\mathbb{Z}_{\geq 0}^{+\infty}$ and $\mathbb{Z}_{\leq 0}^{-\infty}$ with crystal structures as follows. Let $y^{+}=\left(\ldots, y_{k}, \ldots, y_{2}, y_{1}\right) \in$ $\mathbb{Z}_{\geq 0}^{+\infty}$ and $y^{-}=\left(y_{0}, y_{-1} \ldots, y_{k} \ldots\right) \in \mathbb{Z}_{\leq 0}^{-\infty}$. For $k \geq 1$, we set

$$
\sigma_{k}^{+}\left(y^{+}\right)=y_{k}+\sum_{j>k}\left\langle\alpha_{i_{j}}, \alpha_{i_{k}}^{\vee}\right\rangle y_{j}
$$

and for $k \leq 0$, we set

$$
\sigma_{k}^{-}\left(y^{-}\right)=-y_{k}-\sum_{j<k}\left\langle\alpha_{i_{j}}, \alpha_{i_{k}}^{\vee}\right\rangle y_{j}
$$


since $y_{j}=0$ for $|j| \gg 0$, we see that $\sigma_{k}^{ \pm}(y)$ is well-defined, and $\sigma_{k}^{ \pm}(y)=0$ for $|k| \gg 0$. For $i \in I$, we set $\sigma_{(i)}^{+}\left(y^{+}\right):=\max \left\{\sigma_{k}^{+}\left(y^{+}\right) \mid k \geq 1, i_{k}=i\right\}$ and $\sigma_{(i)}^{-}\left(y^{-}\right):=\max \left\{\sigma_{k}^{-}\left(y^{-}\right) \mid k \leq\right.$ $\left.0, i_{k}=i\right\}$, and define

$$
\begin{aligned}
& M_{(i)}^{+}=M_{(i)}^{+}\left(y^{+}\right):=\left\{k \mid k \geq 1, i_{k}=i, \sigma_{k}^{+}\left(y^{+}\right)=\sigma_{(i)}^{+}\left(y^{+}\right)\right\}, \\
& M_{(i)}^{-}=M_{(i)}^{-}\left(y^{-}\right):=\left\{k \mid k \leq 0, i_{k}=i, \sigma_{k}^{-}\left(y^{-}\right)=\sigma_{(i)}^{-}\left(y^{-}\right)\right\} .
\end{aligned}
$$

Note that $\sigma_{(i)}^{ \pm}\left(y^{ \pm}\right) \geq 0$, and that $M_{(i)}^{ \pm}=M_{(i)}^{ \pm}\left(y^{ \pm}\right)$is a finite set if and only if $\sigma_{(i)}^{ \pm}\left(y^{ \pm}\right)>0$. We define the maps $\tilde{e}_{i}, \tilde{f}_{i}: \mathbb{Z}^{+\infty} \rightarrow \mathbb{Z}^{+\infty} \sqcup\{\mathbf{0}\}$ and $\tilde{e}_{i}, \tilde{f}_{i}: \mathbb{Z}^{-\infty} \rightarrow \mathbb{Z}^{-\infty} \sqcup\{\mathbf{0}\}$ by

$$
\begin{aligned}
& \tilde{e}_{i} y^{+}:= \begin{cases}\left(\ldots, y_{k}^{\prime}, \ldots, y_{2}^{\prime}, y_{1}^{\prime}\right) \text { with } y_{k}^{\prime}:=y_{k}-\delta_{k, \max M_{(i)}^{+}} & \text {if } \sigma_{(i)}^{+}\left(y^{+}\right)>0, \\
\mathbf{0} & \text { if } \sigma_{(i)}^{+}\left(y^{+}\right)=0,\end{cases} \\
& \tilde{f}_{i} y^{+}:=\left(\ldots, y_{k}^{\prime}, \ldots, y_{2}^{\prime}, y_{1}^{\prime}\right) \text { with } y_{k}^{\prime}:=y_{k}+\delta_{k, \min M_{(i)}^{+},} \\
& \tilde{e}_{i} y^{-}:=\left(y_{0}^{\prime}, y_{-1}^{\prime} \ldots, y_{k}^{\prime} \ldots\right) \text { with } y_{k}^{\prime}:=y_{k}-\delta_{k, \max M_{(i)}^{-},} \\
& \tilde{f}_{i} y^{-}:= \begin{cases}\left(y_{0}^{\prime}, y_{-1}^{\prime} \ldots, y_{k}^{\prime} \ldots\right) \text { with } y_{k}^{\prime}:=y_{k}+\delta_{k, \min M_{(i)}^{-}} & \text {if } \sigma_{(i)}^{-}\left(y^{-}\right)>0, \\
0 & \text { if } \sigma_{(i)}^{-}\left(y^{-}\right)=0,\end{cases}
\end{aligned}
$$

respectively. Moreover, we define

$$
\begin{aligned}
& \operatorname{wt}\left(y^{+}\right):=-\sum_{j \geq 1} y_{j} \alpha_{i_{j}}, \quad \varepsilon_{i}\left(y^{+}\right):=\sigma_{(i)}^{+}\left(y^{+}\right), \quad \varphi_{i}\left(y^{+}\right):=\varepsilon_{i}\left(y^{+}\right)+\left\langle\operatorname{wt}\left(y^{+}\right), \alpha_{i}^{\vee}\right\rangle, \\
& \operatorname{wt}\left(y^{-}\right):=-\sum_{j \leq 0} y_{j} \alpha_{i_{j}}, \quad \varphi_{i}\left(y^{-}\right):=\sigma_{(i)}^{-}\left(y^{-}\right), \quad \varepsilon_{i}\left(y^{-}\right):=\varphi_{i}\left(y^{-}\right)-\left\langle\operatorname{wt}\left(y^{-}\right), \alpha_{i}^{\vee}\right\rangle .
\end{aligned}
$$

These maps make $\mathbb{Z}_{\geq 0}^{+\infty}$ (resp., $\mathbb{Z}_{\leq 0}^{-\infty}$ ) into a crystal for $\mathfrak{g}$; we denote this crystal by $\mathbb{Z}_{\iota^{+}}^{+\infty}$ (resp., $\mathbb{Z}_{\iota^{-}}^{-\infty}$ ).

Theorem 2.10 ([11, Theorem 2.5]). There exists an embedding $\Psi_{\iota^{+}}^{+}: \mathcal{B}(\infty) \hookrightarrow \mathbb{Z}_{\iota^{+}}^{+\infty}$ of crystals which sends $u_{\infty} \in \mathcal{B}(\infty)$ to $(\ldots, 0 \ldots, 0,0) \in \mathbb{Z}_{\iota^{+}}^{+\infty}$. Similarly, there exists an embedding $\Psi_{\iota^{-}}^{-}: \mathcal{B}(-\infty) \hookrightarrow \mathbb{Z}_{\iota^{-}}^{-\infty}$ of crystals which sends $u_{-\infty} \in \mathcal{B}(-\infty)$ to $(0,0, \ldots, 0, \ldots) \in \mathbb{Z}_{\iota^{-}}^{-\infty}$.

The next corollary follows immediately from Theorem 2.10

Corollary 2.11. For each $\mu \in P$, there exists an embedding $\mathcal{B}(\infty) \otimes \mathcal{T}_{\mu} \otimes \mathcal{B}(-\infty) \hookrightarrow$ $\mathbb{Z}_{\iota^{+}}^{+\infty} \otimes \mathcal{T}_{\mu} \otimes \mathbb{Z}_{\iota^{-}}^{-\infty}$ of crystals which sends $u_{\infty} \otimes t_{\mu} \otimes u_{-\infty} \in \mathcal{B}(\infty) \otimes \mathcal{T}_{\mu} \otimes \mathcal{B}(-\infty)$ to $(\ldots, 0, \ldots, 0,0) \otimes t_{\mu} \otimes(0,0, \ldots, 0, \ldots) \in \mathbb{Z}_{\iota^{+}}^{+\infty} \otimes \mathcal{T}_{\mu} \otimes \mathbb{Z}_{\iota^{-}}^{-\infty}$.

We define $*$-operations on $\operatorname{Im}\left(\Psi_{\iota^{ \pm}}^{ \pm}\right)$and $\operatorname{Im}\left(\Psi_{\iota^{+}}^{+}\right) \otimes \mathcal{T}_{\mu} \otimes \operatorname{Im}\left(\Psi_{\iota^{-}}^{-}\right)$by the following commutative diagrams, respectively:

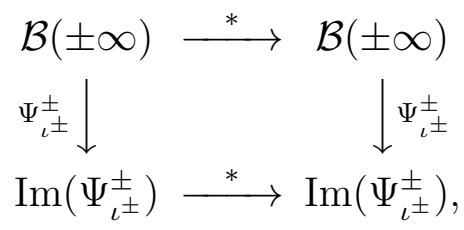




$$
\begin{aligned}
& \mathcal{B}(\infty) \otimes \mathcal{T}_{\mu} \otimes \mathcal{B}(-\infty) \quad{ }^{*} \longrightarrow \mathcal{B}(\infty) \otimes \mathcal{T}_{\mu} \otimes \mathcal{B}(-\infty) \\
& \Psi_{\iota^{+}}^{+} \otimes \mathrm{id} \otimes \Psi_{\iota^{-}}^{-} \downarrow \quad \Psi_{\iota^{+}}^{+} \otimes \mathrm{id} \otimes \Psi_{\iota^{-}}^{-} \\
& \operatorname{Im}\left(\Psi_{\iota^{+}}^{+}\right) \otimes \mathcal{T}_{\mu} \otimes \operatorname{Im}\left(\Psi_{\iota^{-}}^{-}\right) \stackrel{*}{\longrightarrow} \operatorname{Im}\left(\Psi_{\iota^{+}}^{+}\right) \otimes \mathcal{T}_{\mu} \otimes \operatorname{Im}\left(\Psi_{\iota^{-}}^{-}\right) .
\end{aligned}
$$

Then we deduce from [11, Remark in §2.4] that if $z_{1}=\left(\ldots, c_{2}, c_{1}\right) \in \operatorname{Im}\left(\Psi_{\iota^{+}}^{+}\right)$, then $z_{1}^{*}=\tilde{f}_{i_{1}}^{c_{1}} \tilde{f}_{i_{2}}^{c_{2}} \ldots(\ldots, 0,0)$. Similarly, we see that if $z_{2}=\left(c_{0}, c_{-1}, \ldots\right) \in \operatorname{Im}\left(\Psi_{\iota^{-}}^{-}\right)$, then $z_{2}^{*}=$ $\tilde{e}_{i_{0}}^{-c_{0}} \tilde{e}_{i_{-1}}^{-c_{-1}} \cdots(0,0, \ldots)$. Moreover, we see by (2.2) that if $z_{1} \in \operatorname{Im}\left(\Psi_{\iota^{+}}^{+}\right)$and $z_{2} \in \operatorname{Im}\left(\Psi_{\iota^{-}}^{-}\right)$, then

$$
\left(z_{1} \otimes t_{\mu} \otimes z_{2}\right)^{*}=z_{1}^{*} \otimes t_{-\mu-w t}\left(z_{1}\right)-\operatorname{wt}\left(z_{2}\right) \otimes z_{2}^{*} .
$$

By the tensor product rule of crystals, we can describe the crystal structure of $\mathbb{Z}_{\iota^{+}}^{+\infty} \otimes$ $\mathcal{T}_{\mu} \otimes \mathbb{Z}_{\iota^{-}}^{-\infty}$ as follows. Let $y=y^{+} \otimes t_{\mu} \otimes y^{-}$with $y^{+}=\left(\ldots, y_{2}, y_{1}\right) \in \mathbb{Z}_{\iota^{+}}^{+\infty}$ and $y^{-}=$ $\left(y_{0}, y_{-1}, \ldots\right) \in \mathbb{Z}_{\iota^{-}}^{-\infty}$. We set

$$
\sigma_{k}(y):= \begin{cases}\sigma_{k}^{+}\left(y^{+}\right) & \text {if } k \geq 1, \\ \sigma_{k}^{-}\left(y^{-}\right)-\left\langle\operatorname{wt}(y), \alpha_{i_{k}}^{\vee}\right\rangle & \text { if } k \leq 0 .\end{cases}
$$

For $i \in I$, we set $\sigma_{(i)}(y):=\max \left\{\sigma_{k}(y) \mid k \in \mathbb{Z}, i_{k}=i\right\}$ and

$$
M_{(i)}=M_{(i)}(y):=\left\{k \mid i_{k}=i, \sigma_{k}(y)=\sigma_{(i)}(y)\right\} .
$$

Then we see that

$$
\begin{gathered}
\operatorname{wt}(y)=\mu-\sum_{j \in \mathbb{Z}} y_{j} \alpha_{i_{j}}, \quad \varepsilon_{i}(y)=\sigma_{(i)}(y), \quad \varphi_{i}(y)=\varepsilon_{i}(y)+\left\langle\operatorname{wt}(y), \alpha_{i}^{\vee}\right\rangle, \\
\tilde{e}_{i} y= \begin{cases}\left(\ldots, y_{2}^{\prime}, y_{1}^{\prime}\right) \otimes t_{\mu} \otimes\left(y_{0}^{\prime}, y_{-1}^{\prime}, \ldots\right) \text { with } y_{k}^{\prime}:=y_{k}-\delta_{k, \max M_{(i)}} & \text { if } \varepsilon_{i}(y)>0, \\
0 & \text { if } \varepsilon_{i}(y)=0,\end{cases} \\
\tilde{f}_{i} y= \begin{cases}\left(\ldots, y_{2}^{\prime}, y_{1}^{\prime}\right) \otimes t_{\mu} \otimes\left(y_{0}^{\prime}, y_{-1}^{\prime}, \ldots\right) \text { with } y_{k}^{\prime}:=y_{k}+\delta_{k, \min M_{(i)}} & \text { if } \varphi_{i}(y)>0, \\
0 & \text { if } \varphi_{i}(y)=0 .\end{cases}
\end{gathered}
$$

\section{Main results.}

In the following, we assume that the generalized Cartan matrix $A$ is of the form

$$
A=\left(\begin{array}{cc}
2 & -a_{1} \\
-a_{2} & 2
\end{array}\right), \text { where } a_{1}, a_{2} \in \mathbb{Z}_{\geq 2} \text { with } a_{1} a_{2}>4
$$

note that $\alpha_{1}=2 \Lambda_{1}-a_{2} \Lambda_{2}$ and $\alpha_{2}=-a_{1} \Lambda_{1}+2 \Lambda_{2}$. We set $P=\mathbb{Z} \Lambda_{1} \oplus \mathbb{Z} \Lambda_{2}$. Let $\lambda \in P$ be an integral weight of the form either (ii) or (iii): 
(i) $\lambda=k_{1} \Lambda_{1}-k_{2} \Lambda_{2}$ for some $k_{1}, k_{2} \in \mathbb{Z}_{>0}$ such that $k_{2} \leq k_{1}<\left(a_{1}-1\right) k_{2}$;

(ii) $\lambda=k_{1} \Lambda_{1}-k_{2} \Lambda_{2}$ for some $k_{1}, k_{2} \in \mathbb{Z}_{>0}$ such that $k_{1}<k_{2} \leq\left(a_{2}-1\right) k_{1}$.

Remark 3.1. Let $\mathbb{O}:=\{W \mu \mid \mu \in P\}$ be the set of $W$-orbits in $P$. We know from [1, Theorem 3.1] that $O \in \mathbb{O}$ satisfies condition (2.1), that is, $O \cap\left(P^{+} \cup-P^{+}\right)=\emptyset$ if and only if $O$ contains an integral weight of the form either (ii) or (iii) above.

Let $\mathbb{B}_{0}(\lambda)$ (resp., $\mathcal{B}_{0}(\lambda)$ ) be the connected component of $\mathbb{B}(\lambda)$ (resp., $\mathcal{B}(\lambda)$ ) containing $\pi_{\lambda}:=(\lambda ; 0,1)\left(\right.$ resp., $\left.u_{\lambda}\right)$.

Theorem 3.2 (will be proved in \$5.1). Let $\lambda$ be an integral weight of the form either (ii) or (iii) above. There exists an isomorphism $\mathbb{B}_{0}(\lambda) \rightarrow \mathcal{B}_{0}(\lambda)$ of crystals that sends $\pi_{\lambda}$ to $u_{\lambda}$.

Theorem 3.3 (will be proved in $\$ 5.2$ ). Assume that $k_{1}=1$ or $k_{2}=1$, that is, $\lambda \in P$ is of the form either $k_{1} \Lambda_{1}-\Lambda_{2}$ with $1 \leq k_{1}<a_{1}-1$ or $\Lambda_{1}-k_{2} \Lambda_{2}$ with $1<k_{2} \leq a_{2}-1$. For $b \in \mathcal{B}(\lambda)$, there exist $i_{1}, \ldots, i_{r} \in I$ such that $b=\tilde{f}_{i_{r}} \cdots \tilde{f}_{i_{1}} u_{\lambda}$ or $b=\tilde{e}_{i_{r}} \cdots \tilde{e}_{i_{1}} u_{\lambda}$. In particular, the crystal graph of $\mathcal{B}(\lambda)$ is connected.

Let $\lambda$ be as in Theorem 3.3. By [1, Theorem 4.1] (resp., Theorem [3.3), we have $\mathbb{B}(\lambda)=\mathbb{B}_{0}(\lambda)$ (resp., $\mathcal{B}(\lambda)=\mathcal{B}_{0}(\lambda)$ ). Therefore, by Theorem 3.2 , we obtain the following corollary.

Corollary 3.4. If $k_{1}=1$ or $k_{2}=1$, then there exists an isomorphism $\mathbb{B}(\lambda) \rightarrow \mathcal{B}(\lambda)$ of crystals that sends $\pi_{\lambda}$ to $u_{\lambda}$.

\section{Rank 2 case.}

\subsection{LS paths in the rank 2 case.}

Let $A$ and $\lambda=k_{1} \Lambda_{1}-k_{2} \Lambda_{2}$ be as in \$3. In this subsection, we recall some properties of $\mathbb{B}(\lambda)$ from [1]. We define the sequence $\left\{p_{m}\right\}_{m \in \mathbb{Z}}$ of integers by the following recursive formulas: for $m \geq 0$,

$$
p_{0}:=k_{2}, \quad p_{1}:=k_{1}, \quad p_{m+2}:= \begin{cases}a_{2} p_{m+1}-p_{m} & \text { if } m \text { is even } \\ a_{1} p_{m+1}-p_{m} & \text { if } m \text { is odd }\end{cases}
$$

for $m<0$,

$$
p_{m}= \begin{cases}a_{2} p_{m+1}-p_{m+2} & \text { if } m \text { is even } \\ a_{1} p_{m+1}-p_{m+2} & \text { if } m \text { is odd }\end{cases}
$$


it follows from [1, Remark 3.7] (and the comment in [15, §3.1]) that

$$
p_{m}>0 \text { for all } m \in \mathbb{Z} \text {. }
$$

Notice that $W=\left\{x_{m} \mid m \in \mathbb{Z}\right\}$, where

$$
x_{m}:= \begin{cases}\left(s_{2} s_{1}\right)^{n} & \text { if } m=2 n \text { with } n \in \mathbb{Z}_{\geq 0}, \\ s_{1}\left(s_{2} s_{1}\right)^{n} & \text { if } m=2 n+1 \text { with } n \in \mathbb{Z}_{\geq 0}, \\ \left(s_{1} s_{2}\right)^{-n} & \text { if } m=2 n \text { with } n \in \mathbb{Z}_{\leq 0}, \\ s_{2}\left(s_{1} s_{2}\right)^{-n} & \text { if } m=2 n-1 \text { with } n \in \mathbb{Z}_{\leq 0} .\end{cases}
$$

Then we have

$$
x_{m} \lambda= \begin{cases}p_{m+1} \Lambda_{1}-p_{m} \Lambda_{2} & \text { if } m \text { is even } \\ -p_{m} \Lambda_{1}+p_{m+1} \Lambda_{2} & \text { if } m \text { is odd }\end{cases}
$$

for $m \in \mathbb{Z}$ by [1, Lemma 3.3].

Proposition 4.1 ([1, Proposition 3.8]). The Hasse diagram of $W \lambda$ in the ordering of Definition 2.5 is

$$
\cdots \stackrel{\alpha_{1}}{\longleftarrow} x_{2} \lambda \stackrel{\alpha_{2}}{\longleftarrow} x_{1} \lambda \stackrel{\alpha_{1}}{\longleftarrow} x_{0} \lambda \stackrel{\alpha_{2}}{\longleftarrow} x_{-1} \lambda \stackrel{\alpha_{1}}{\longleftarrow} x_{-2} \lambda \stackrel{\alpha_{2}}{\longleftarrow} \cdots
$$

For each $\nu \in W \lambda$, there exists a unique $m \in \mathbb{Z}$ such that $\nu=x_{m} \lambda$. Then we define $z(\nu):=m$. We set

$$
\mathbb{B}_{1}(\lambda):=\left\{\left(\nu_{1}, \ldots, \nu_{u} ; \sigma_{1}, \ldots, \sigma_{u}\right) \in \mathbb{B}(\lambda) \mid z\left(\nu_{v}\right)-z\left(\nu_{v+1}\right)=1 \text { for } v=1, \ldots, u-1\right\}
$$

note that $\pi_{\lambda}=(\lambda ; 0,1) \in \mathbb{B}_{1}(\lambda)$. We know from [1, Theorem 4.17] that $\mathbb{B}_{1}(\lambda) \cup\{\mathbf{0}\}$ is stable under the action of $\tilde{e}_{i}, \tilde{f}_{i}$ for $i \in I=\{1,2\}$. Hence, $\mathbb{B}_{1}(\lambda)$ is a subcrystal of $\mathbb{B}(\lambda)$ (but not necessarily, a connected component of $\mathbb{B}(\lambda)$ ). Since $\mathbb{B}_{0}(\lambda)$ is the connected component of $\mathbb{B}(\lambda)$ containing $\pi_{\lambda}$, by the definition, it follows that

$$
\mathbb{B}_{0}(\lambda) \subset \mathbb{B}_{1}(\lambda)
$$

We deduce by (4.3) and (4.4) that an element $\pi \in \mathbb{B}_{1}(\lambda)$ is of the form

$$
\pi=\left(x_{m} \lambda, x_{m-1} \lambda, \ldots, x_{n} \lambda ; 0, \frac{q_{m}}{p_{m}}, \frac{q_{m-1}}{p_{m-1}}, \ldots, \frac{q_{n+1}}{p_{n+1}}, 1\right)
$$

where $n \leq m$, and $q_{m}, q_{m-1}, \ldots, q_{n+1}$ are integers satisfying

$$
0<q_{j}<p_{j} \text { for } n+1 \leq j \leq m, \text { and } \frac{q_{j+1}}{p_{j+1}}<\frac{q_{j}}{p_{j}} \text { for } n+1 \leq j \leq m-1 \text {. }
$$


Remark 4.2. Let $\pi=\left(x_{n+s-1} \lambda, \ldots, x_{n+1} \lambda, x_{n} \lambda ; \sigma_{0}, \sigma_{1}, \ldots, \sigma_{s}\right) \in \mathbb{B}_{1}(\lambda)$, and $i \in I=$ $\{1,2\}$. We see by (4.3), (4.4), and the definition of $\mathbb{B}_{1}(\lambda)$ that the function $H_{i}^{\pi}(t)=$ $\left\langle\pi(t), \alpha_{i}^{\vee}\right\rangle$ attains either a maximal value or a minimal value at $t \in[0,1]$ if and only if $t \in\left\{0=\sigma_{0}, \sigma_{1}, \ldots, \sigma_{s}=1\right\}$. Moreover, if $H_{i}^{\pi}(t)$ attains a minimal (resp., maximal) value at $t=\sigma_{v}$, then $H_{i}^{\pi}(t)$ attains a minimal (resp., maximal) value at $t=\sigma_{u}$ for all $u=0,1, \ldots, s$ such that $u \equiv v \bmod 2$.

Remark 4.3. Assume that $k_{1}$ and $k_{2}$ are relatively prime. We know from [1, Lemma 4.5 (3)] that an LS path of shape $\lambda=k_{1} \Lambda_{1}-k_{2} \Lambda_{2}$ is of the form (4.6). Hence we have $\mathbb{B}_{1}(\lambda)=\mathbb{B}(\lambda)$.

Theorem 4.4 ([1, Theorem 4.1]). If $k_{1}=1$ or $k_{2}=1$, that is, $\lambda \in P$ is of the form either $k_{1} \Lambda_{1}-\Lambda_{2}$ with $1 \leq k_{1}<a_{1}-1$ or $\Lambda_{1}-k_{2} \Lambda_{2}$ with $1<k_{2} \leq a_{2}-1$, then the crystal graph of $\mathbb{B}(\lambda)$ is connected. Otherwise, the crystal graph of $\mathbb{B}(\lambda)$ has infinitely many connected components.

\subsection{Polyhedral realizations of $\mathcal{B}( \pm \infty)$ in the rank 2 case.}

Let $A$ and $\lambda=k_{1} \Lambda_{1}-k_{2} \Lambda_{2}$ be as in $\$ 3$. Set $\iota^{+}:=(\ldots, 2,1,2,1)$ and $\iota^{-}:=(2,1,2,1, \ldots)$. We define the sequence $\left\{c_{j}\right\}_{j \in \mathbb{Z}}$ of integers by the following recursive formulas: for $j \geq 1$,

$$
c_{1}:=1, \quad c_{2}:=a_{1}, \quad c_{j+2}:= \begin{cases}a_{1} c_{j+1}-c_{j} & \text { if } j \text { is even } \\ a_{2} c_{j+1}-c_{j} & \text { if } j \text { is odd }\end{cases}
$$

for $j \leq 0$,

$$
c_{0}:=1, \quad c_{-1}:=a_{2}, \quad c_{j-2}:= \begin{cases}a_{1} c_{j-1}-c_{j} & \text { if } j \text { is even } \\ a_{2} c_{j-1}-c_{j} & \text { if } j \text { is odd }\end{cases}
$$

Applying [11, Theorem 4.1] to our rank 2 case, we obtain the following explicit description of the image of $\Psi_{\iota^{+}}^{+}$and $\Psi_{\iota^{-}}^{-}$.

Proposition 4.5. It hold that

$$
\begin{aligned}
& \operatorname{Im}\left(\Psi_{\iota^{+}}^{+}\right)=\left\{\left(\ldots, y_{2}, y_{1}\right) \in \mathbb{Z}_{\geq 0}^{+\infty} \mid c_{l} y_{l}-c_{l-1} y_{l+1} \geq 0 \text { for } l \geq 2\right\} \\
& \operatorname{Im}\left(\Psi_{\iota^{-}}^{-}\right)=\left\{\left(y_{0}, y_{-1}, \ldots\right) \in \mathbb{Z}_{\leq 0}^{-\infty} \mid c_{l} y_{l}-c_{l+1} y_{l-1} \leq 0 \text { for } l \leq-1\right\} .
\end{aligned}
$$

The following lemma will be needed in the next section. Recall that $\left\{p_{m}\right\}_{m \in \mathbb{Z}}$ is defined by the recursive formulas (4.1) and (4.2).

Lemma 4.6. Let $m, n \in \mathbb{Z}$ be such that $n<m$ and let $q_{n+1}, q_{n+2}, \ldots, q_{m} \in \mathbb{Z}$ be such that $0<q_{j}<p_{j}$ for $n+1 \leq j \leq m$, and $q_{j+1} / p_{j+1}<q_{j} / p_{j}$ for $n+1 \leq j \leq m-1$.

(1) If $0<m$, then $\left(\ldots, 0, p_{m}, \ldots, p_{2}, p_{1}\right) \in \operatorname{Im}\left(\Psi_{\iota^{+}}^{+}\right)$. 
(2) If $0<n<m$, then $\left(\ldots, 0, q_{m}, \ldots, q_{n+2}, q_{n+1}, p_{n}, \ldots, p_{2}, p_{1}\right) \in \operatorname{Im}\left(\Psi_{\iota^{+}}^{+}\right)$.

(3) If $0<m$, then $\left(\ldots, 0, q_{m}, \ldots, q_{2}, q_{1}\right) \in \operatorname{Im}\left(\Psi_{\iota^{-}}^{+}\right)$.

(4) If $n<0$, then $\left(q_{0}-p_{0}, q_{-1}-p_{-1}, \ldots, q_{n+1}-p_{n+1}, 0, \ldots\right) \in \operatorname{Im}\left(\Psi_{\iota^{-}}^{-}\right)$.

(5) If $n<m<0$, then $\left(-p_{0},-p_{-1}, \ldots,-p_{m+1}, q_{m}-p_{m}, \ldots, q_{n+1}-p_{n+1}, 0, \ldots\right) \in \operatorname{Im}\left(\Psi_{\iota^{-}}^{-}\right)$.

(6) If $n<0$, then $\left(-p_{0},-p_{-1}, \ldots,-p_{n+1}, 0, \ldots\right) \in \operatorname{Im}\left(\Psi_{\iota^{-}}^{-}\right)$.

Proof. We give proofs only for parts (2) and (5); the proof for the other cases is easier than these cases.

First, we show part (2). By Proposition 4.5, it suffices to show that

$$
\begin{array}{ll}
c_{j} p_{j}-c_{j-1} p_{j+1} \geq 0 & \text { for } 2 \leq j \leq n-1, \\
c_{j} p_{j}-c_{j-1} q_{j+1} \geq 0 & \text { for } j=n, \\
c_{j} q_{j}-c_{j-1} q_{j+1} \geq 0 & \text { for } n+1 \leq j \leq m-1 .
\end{array}
$$

We can easily see by induction on $j$ that

$$
c_{j} p_{j}-c_{j-1} p_{j+1} \geq 0 \quad \text { for } j \geq 2 \text {. }
$$

Thus we get (4.7). Since $q_{n+1} / p_{n+1}<1$, we see that $c_{n} p_{n}-c_{n-1} q_{n+1}>c_{n} p_{n}-c_{n-1} p_{n+1}$. Combining this inequality and (4.10), we obtain (4.8). For $n+1 \leq j \leq m-1$, we see that $c_{j} q_{j}-c_{j-1} q_{j+1}>c_{j}\left(q_{j+1} p_{j} / p_{j+1}\right)-c_{j-1} q_{j+1}=\left(q_{j+1} / p_{j+1}\right)\left(c_{j} p_{j}-c_{j-1} p_{j+1}\right)$ since $q_{j+1} / p_{j+1}<q_{j} / p_{j}$. Combining this inequality and (4.10), we obtain (4.9). Thus we have proved part (2).

Next, we show part (5). Similarly, it suffices to show that

$$
\begin{aligned}
& c_{j}\left(-p_{j}\right)-c_{j+1}\left(-p_{j-1}\right) \leq 0 \text { for } m+2 \leq j \leq-1, \\
& c_{j}\left(-p_{j}\right)-c_{j+1}\left(q_{j-1}-p_{j-1}\right) \leq 0 \quad \text { for } j=m+1, \\
& c_{j}\left(q_{j}-p_{j}\right)-c_{j+1}\left(q_{j-1}-p_{j+1}\right) \leq 0 \quad \text { for } n+2 \leq j \leq m .
\end{aligned}
$$

We can easily see by induction on $j$ that

$$
-c_{j} p_{j}+c_{j+1} p_{j-1} \leq 0 \quad \text { for } j \leq-1 .
$$

Thus we get (4.11). We see that $c_{m+1}\left(-p_{m+1}\right)-c_{m+2}\left(q_{m}-p_{m}\right)=-c_{m+1} p_{m+1}+c_{m+2} p_{m}-$ $c_{m+2} q_{m}$. Combining this equality and (4.14), we obtain (4.12). For $n+2 \leq j \leq m$, we see that

$$
\begin{aligned}
c_{j}\left(q_{j}-p_{j}\right)-c_{j+1}\left(q_{j-1}-p_{j-1}\right) & =c_{j} q_{j}-c_{j+1} q_{j-1}-c_{j} p_{j}+c_{j-1} p_{j-1} \\
& <c_{j}\left(\frac{q_{j-1} p_{j}}{p_{j-1}}\right)-c_{j+1} q_{j-1}+\left(-c_{j} p_{j}+c_{j+1} p_{j-1}\right) \\
& =\left(1-\frac{q_{j-1}}{p_{j-1}}\right)\left(-c_{j} p_{j}+c_{j+1} p_{j-1}\right)
\end{aligned}
$$


since $q_{j} / p_{j}<q_{j-1} / p_{j-1}$. Combining this inequality and (4.14), we obtain (4.13). Thus we have proved part (5) .

\section{Proofs.}

\subsection{Proof of Theorem 3.2 .}

Let $\iota:=\left(\iota^{+}, \iota^{-}\right)=((\ldots, 2,1,2,1),(2,1,2,1, \ldots))$, and let $\lambda=k_{1} \Lambda_{1}-k_{2} \Lambda_{2}$ be as in $\$ 3$, We define a map $\Phi_{\iota}^{\lambda}$ from $\mathbb{B}_{1}(\lambda) \cup\{\mathbf{0}\}$ to $\operatorname{Im}\left(\Psi_{\iota^{+}}^{+}\right) \otimes \mathcal{T}_{\lambda} \otimes \operatorname{Im}\left(\Psi_{\iota^{-}}^{-}\right) \cup\{\mathbf{0}\}$ as follows. First, we set $\Phi_{\iota}^{\lambda}(\mathbf{0}):=\mathbf{0}$. Let

$$
\pi=\left(x_{m} \lambda, x_{m-1} \lambda, \ldots, x_{n} \lambda ; 0, \frac{q_{m}}{p_{m}}, \frac{q_{m-1}}{p_{m-1}}, \ldots, \frac{q_{n+1}}{p_{n+1}}, 1\right) \in \mathbb{B}_{1}(\lambda),
$$

where $n \leq m$, and $q_{m}, q_{m-1}, \ldots, q_{n+1}$ are integers satisfying $0<q_{j}<p_{j}$ for $n+1 \leq j \leq m$ and $q_{j+1} / p_{j+1}<q_{j} / p_{j}$ for $n+1 \leq j \leq m-1$. We set

$$
z_{k}=z_{k}(\pi):= \begin{cases}q_{k} & \text { if } 1 \leq k \text { and } n+1 \leq k \leq m \\ p_{k} & \text { if } 1 \leq k \text { and } k \leq n \\ q_{k}-p_{k} & \text { if } k \leq 0 \text { and } n+1 \leq k \leq m \\ -p_{k} & \text { if } k \leq 0 \text { and } m+1 \leq k \\ 0 & \text { otherwise }\end{cases}
$$

for $k \in \mathbb{Z}$, and then define $\Phi_{\iota}^{\lambda}(\pi):=\left(\ldots, z_{2}, z_{1}\right) \otimes t_{\lambda} \otimes\left(z_{0}, z_{-1}, \ldots\right) \in \mathbb{Z}_{\iota^{+}}^{+\infty} \otimes \mathcal{T}_{\lambda} \otimes \mathbb{Z}_{\iota^{-}}^{-\infty}$.

Remark 5.1. More explicitly, we can describe $\Phi_{\iota}^{\lambda}(\pi)$ as follows:

(i) if $n=m=0$, that is, $\pi=\pi_{\lambda}$, then $\Phi_{\iota}^{\lambda}(\pi)=(\ldots, 0,0) \otimes t_{\lambda} \otimes(0,0, \ldots)$;

(ii) if $0<n=m$, then $\Phi_{\iota}^{\lambda}(\pi)=\left(\ldots, 0, p_{m}, \ldots, p_{2}, p_{1}\right) \otimes t_{\lambda} \otimes(0,0, \ldots)$;

(iii) if $0<n<m$, then $\Phi_{\iota}^{\lambda}(\pi)=\left(\ldots, 0, q_{m}, \ldots, q_{n+2}, q_{n+1}, p_{n}, \ldots, p_{2}, p_{1}\right) \otimes t_{\lambda} \otimes(0,0, \ldots)$;

(iv) if $n=0<m$, then $\Phi_{\iota}^{\lambda}(\pi)=\left(\ldots, 0, q_{m}, \ldots, q_{2}, q_{1}\right) \otimes t_{\lambda} \otimes(0,0, \ldots)$;

$(\mathrm{v})$ if $n<0<m$, then $\Phi_{\iota}^{\lambda}(\pi)=\left(\ldots, 0, q_{m}, \ldots, q_{2}, q_{1}\right) \otimes t_{\lambda} \otimes\left(q_{0}-p_{0}, q_{-1}-p_{-1}, \ldots, q_{n+1}-\right.$ $\left.p_{n+1}, 0, \ldots\right)$;

(vi) if $n<m=0$, then $\Phi_{\iota}^{\lambda}(\pi)=(\ldots, 0,0) \otimes t_{\lambda} \otimes\left(q_{0}-p_{0}, q_{-1}-p_{-1}, \ldots, q_{n+1}-p_{n+1}, 0, \ldots\right)$;

(vii) if $n<m<0$, then $\Phi_{\iota}^{\lambda}(\pi)=(\ldots, 0,0) \otimes t_{\lambda} \otimes\left(-p_{0},-p_{-1}, \ldots,-p_{m+1}, q_{m}-p_{m}, q_{m-1}-\right.$ $\left.p_{m-1}, \ldots, q_{n+1}-p_{n+1}, 0, \ldots\right)$;

(viii) if $n=m<0$, then $\Phi_{\iota}^{\lambda}(\pi)=(\ldots, 0,0) \otimes t_{\lambda} \otimes\left(-p_{0},-p_{-1}, \ldots,-p_{n+1}, 0, \ldots\right)$. 
Therefore, by Lemma 4.6, we deduce that $\Phi_{\iota}^{\lambda}(\pi) \in \operatorname{Im}\left(\Psi_{\iota^{+}}^{+}\right) \otimes \mathcal{T}_{\lambda} \otimes \operatorname{Im}\left(\Psi_{\iota^{-}}^{-}\right)$for $\pi \in \mathbb{B}_{1}(\lambda)$.

Theorem 5.2. The map $\Phi_{\iota}^{\lambda}: \mathbb{B}_{1}(\lambda) \rightarrow \operatorname{Im}\left(\Psi_{\iota^{+}}^{+}\right) \otimes \mathcal{T}_{\lambda} \otimes \operatorname{Im}\left(\Psi_{\iota^{-}}^{-}\right)$is an embedding of crystals.

Assuming that Theorem 5.2 is true, we give a proof of Theorem 3.2 .

Proof of Theorem 3.2. Let $Z(\lambda):=\left\{b \in \operatorname{Im}\left(\Psi_{\iota^{+}}^{+}\right) \otimes \mathcal{T}_{\lambda} \otimes \operatorname{Im}\left(\Psi_{\iota^{-}}^{-}\right) \mid b^{*}\right.$ is extremal $\}$. We know from Theorem 2.4 and Corollary 2.11 that there exists an isomorphism $\Sigma: Z(\lambda) \rightarrow$ $\mathcal{B}(\lambda)$, which sends $z_{\lambda}:=(\ldots, 0,0) \otimes t_{\lambda} \otimes(0,0, \ldots)$ to $u_{\lambda}$. Recall from (4.5) that $\mathbb{B}_{0}(\lambda) \subset$ $\mathbb{B}_{1}(\lambda)$. Because $\Phi_{\iota}^{\lambda}\left(\pi_{\lambda}\right)=z_{\lambda} \in Z(\lambda)$, we see that $\Phi_{\iota}^{\lambda}\left(\mathbb{B}_{0}(\lambda)\right) \subset Z(\lambda)$. Therefore it follows from Theorem 5.2 that $\left.\Sigma \circ \Phi_{\iota}^{\lambda}\right|_{\mathbb{B}_{0}(\lambda)}$ is an isomorphism of crystals from $\mathbb{B}_{0}(\lambda)$ onto $\mathcal{B}_{0}(\lambda)$. Thus we have proved Theorem 3.2 .

The rest of this subsection is devoted to a proof of Theorem 5.2 .

Lemma 5.3. For $k \leq l$, it holds that

$$
x_{k} \lambda-x_{l} \lambda=\sum_{j=k+1}^{l} p_{j} \alpha_{i_{j}}
$$

Proof. We proceed by induction on $l$; recall that $l \geq k$. If $l=k$, then the assertion is obvious. Assume that $l>k$. By the induction hypothesis, we have $x_{k} \lambda-x_{l-1} \lambda=$ $\sum_{j=k+1}^{l-1} p_{j} \alpha_{i_{j}}$. We see by (4.4) that $x_{l} \lambda=x_{l-1} \lambda-p_{l} \alpha_{i_{l}}$. Therefore, we obtain

$$
x_{k} \lambda-x_{l} \lambda=x_{k} \lambda-x_{l-1} \lambda+p_{l} \alpha_{i_{l}}=\sum_{j=k+1}^{l-1} p_{j} \alpha_{i_{j}}+p_{l} \alpha_{i_{l}}=\sum_{j=k+1}^{l} p_{j} \alpha_{i_{j}},
$$

as desired.

Proposition 5.4. Let $\pi \in \mathbb{B}_{1}(\lambda)$ be as (5.1). Then,

$$
\mathrm{wt}(\pi)=\mathrm{wt}\left(\Phi_{\iota}^{\lambda}(\pi)\right)=x_{n} \lambda-\sum_{j=n+1}^{m} q_{j} \alpha_{i_{j}} .
$$

Proof. First, we show by induction on $m$ that $\operatorname{wt}(\pi)=x_{n} \lambda-\sum_{j=n+1}^{m} q_{j} \alpha_{i_{j}}$; recall that $m \geq n$. If $m=n$, then $\operatorname{wt}(\pi)=x_{n} \lambda$ since $\pi=\left(x_{n} \lambda ; 0,1\right)$. Hence the assertion is obvious. Assume that $m>n$. We see that

$$
\pi^{\prime}:=\left(x_{m-1} \lambda, x_{m-2} \lambda, \ldots, x_{n} \lambda ; 0, \frac{q_{m-1}}{p_{m-1}}, \frac{q_{m-2}}{p_{m-2}}, \ldots, \frac{q_{n+1}}{p_{n+1}}, 1\right)
$$


is also an element of $\mathbb{B}_{1}(\lambda)$. By the induction hypothesis, we obtain $\operatorname{wt}\left(\pi^{\prime}\right)=x_{n} \lambda-$ $\sum_{j=n+1}^{m-1} q_{j} \alpha_{i_{j}}$. We see by the definition of wt that

$$
\begin{aligned}
\operatorname{wt}(\pi) & =\operatorname{wt}\left(\pi^{\prime}\right)-\frac{q_{m-1}}{p_{m-1}} x_{m-1} \lambda+\frac{q_{m}}{p_{m}} x_{m} \lambda+\left(\frac{q_{m-1}}{p_{m-1}}-\frac{q_{m}}{p_{m}}\right) x_{m-1} \lambda \\
& =\operatorname{wt}\left(\pi^{\prime}\right)+\frac{q_{m}}{p_{m}}\left(x_{m} \lambda-x_{m-1} \lambda\right) .
\end{aligned}
$$

We see from Lemma 5.3 that $x_{m} \lambda-x_{m-1} \lambda=-p_{m} \alpha_{i_{m}}$. Therefore we deduce that $\operatorname{wt}(\pi)=$ $x_{n} \lambda-\sum_{j=n+1}^{m-1} q_{j} \alpha_{i_{j}}+\left(q_{m} / p_{m}\right)\left(-p_{m} \alpha_{i_{m}}\right)=x_{n} \lambda-\sum_{j=n+1}^{m} q_{j} \alpha_{i_{j}}$.

Next, we show that $\operatorname{wt}\left(\Phi_{\iota}^{\lambda}(\pi)\right)=x_{n} \lambda-\sum_{j=n+1}^{m} q_{j} \alpha_{i_{j}}$. By the definition of wt, if $0 \leq n \leq m$, then we have

$$
\operatorname{wt}\left(\Phi_{\iota}^{\lambda}(\pi)\right)=\lambda-\sum_{j=n+1}^{m} q_{j} \alpha_{i_{j}}-\sum_{j=1}^{n} p_{j} \alpha_{i_{j}}
$$

if $n<0<m$, then we have

$$
\operatorname{wt}\left(\Phi_{\iota}^{\lambda}(\pi)\right)=\lambda-\sum_{j=1}^{m} q_{j} \alpha_{i_{j}}-\sum_{j=n+1}^{0}\left(q_{j}-p_{j}\right) \alpha_{i_{j}}=\lambda-\sum_{j=n+1}^{m} q_{j} \alpha_{i_{j}}+\sum_{j=n+1}^{0} p_{j} \alpha_{i_{j}}
$$

if $n \leq m \leq 0$, then we have

$$
\operatorname{wt}\left(\Phi_{\iota}^{\lambda}(\pi)\right)=\lambda-\sum_{j=m+1}^{0}\left(-p_{j}\right) \alpha_{i_{j}}-\sum_{j=n+1}^{m}\left(q_{j}-p_{j}\right) \alpha_{i_{j}}=\lambda+\sum_{j=n+1}^{0} p_{j} \alpha_{i_{j}}-\sum_{j=n+1}^{m} q_{j} \alpha_{i_{j}} .
$$

It follows from Lemma 5.3 that

$$
x_{n} \lambda= \begin{cases}\lambda-\sum_{j=1}^{n} p_{j} \alpha_{i_{j}} & \text { if } n \geq 0 \\ \lambda+\sum_{j=n+1}^{0} p_{j} \alpha_{i_{j}} & \text { if } n \leq 0 .\end{cases}
$$

Therefore we obtain $\operatorname{wt}\left(\Phi_{\iota}^{\lambda}(\pi)\right)=x_{n} \lambda-\sum_{j=n+1}^{m} q_{j} \alpha_{i_{j}}$ for $n, m \in \mathbb{Z}$ such that $n \leq m$. Thus we have proved the proposition.

Lemma 5.5. Let $\pi \in \mathbb{B}_{1}(\lambda)$ be as (5.1). Then, for $k \in \mathbb{Z}$,

$$
\sigma_{k}\left(\Phi_{\iota}^{\lambda}(\pi)\right)= \begin{cases}0 & \text { if } m+1 \leq k, \\ q_{k}+\sum_{j=k+1}^{m}\left\langle\alpha_{i_{j}}, \alpha_{i_{k}}^{\vee}\right\rangle q_{j} & \text { if } n+1 \leq k \leq m, \\ -\left\langle\operatorname{wt}\left(\Phi_{\iota}^{\lambda}(\pi)\right), \alpha_{i_{k}}^{\vee}\right\rangle & \text { if } k \leq n\end{cases}
$$

Proof. First, we assume that $0 \leq n \leq m$. We write $\Phi_{\iota}^{\lambda}(\pi)=b_{1} \otimes t_{\lambda} \otimes(0,0, \ldots)$ with $b_{1}=$ $\left(\ldots, 0, q_{m}, \ldots, q_{n+2}, q_{n+1}, p_{n}, \ldots, p_{2}, p_{1}\right)$, where we understand $b_{1}=\left(\ldots, 0, p_{m}, \ldots, p_{2}, p_{1}\right)$ (resp., $\left(\ldots, 0, q_{m}, \ldots, q_{2}, q_{1}\right)$ ) if $0<n=m$ (resp., $n=0<m$ ). If $n+1 \leq k$, then we have $\sigma_{k}\left(\Phi_{\iota}^{\lambda}(\pi)\right)=\sigma_{k}^{+}\left(b_{1}\right)$. Hence the assertion is obvious by the definition of $\sigma_{k}^{+}$. 
Assume that $1 \leq k \leq n$. By Proposition 5.4, it suffices to show that $\sigma_{k}\left(\Phi_{\iota}^{\lambda}(\pi)\right)=$ $-\left\langle x_{n} \lambda, \alpha_{i_{k}}^{\vee}\right\rangle+\sum_{j=n+1}^{m}\left\langle\alpha_{i_{j}}, \alpha_{i_{k}}^{\vee}\right\rangle q_{j}$. We see by the definition of $\sigma_{k}$ that

$$
\sigma_{k}\left(\Phi_{\iota}^{\lambda}(\pi)\right)=\sigma_{k}^{+}\left(b_{1}\right)=p_{k}+\sum_{j=k+1}^{n}\left\langle\alpha_{i_{j}}, \alpha_{i_{k}}^{\vee}\right\rangle p_{j}+\sum_{j=n+1}^{m}\left\langle\alpha_{i_{j}}, \alpha_{i_{k}}^{\vee}\right\rangle q_{j} .
$$

It follows from Lemma 5.3 that $x_{k} \lambda-x_{n} \lambda=\sum_{j=k+1}^{n} p_{j} \alpha_{i_{j}}$. Therefore,

$$
-\left\langle x_{n} \lambda, \alpha_{i_{k}}^{\vee}\right\rangle=-\left\langle x_{k} \lambda, \alpha_{i_{k}}^{\vee}\right\rangle+\sum_{j=k+1}^{n}\left\langle\alpha_{i_{j}}, \alpha_{i_{k}}^{\vee}\right\rangle p_{j}=p_{k}+\sum_{j=k+1}^{n}\left\langle\alpha_{i_{j}}, \alpha_{i_{k}}^{\vee}\right\rangle p_{j}
$$

since $\left\langle x_{k} \lambda, \alpha_{i_{k}}^{\vee}\right\rangle=-p_{k}$ by (4.4). Combining (5.3) and (5.4), we obtain the desired equality. If $k \leq 0$, then we have $\sigma_{k}\left(\Phi_{\iota}^{\lambda}(\pi)\right)=\sigma_{k}^{-}((0,0, \ldots))-\left\langle\operatorname{wt}\left(\Phi_{\iota}^{\lambda}(\pi)\right), \alpha_{i_{k}}^{\vee}\right\rangle=-\left\langle\operatorname{wt}\left(\Phi_{\iota}^{\lambda}(\pi)\right), \alpha_{i_{k}}^{\vee}\right\rangle$.

Next, we assume that $n<0<m$. We write $\Phi_{\iota}^{\lambda}(\pi)=b_{1} \otimes t_{\lambda} \otimes b_{2}$ with $b_{1}=$ $\left(\ldots, 0, q_{m}, \ldots, q_{2}, q_{1}\right)$ and $b_{2}=\left(q_{0}-p_{0}, q_{-1}-p_{-1}, \ldots, q_{n+1}-p_{n+1}, 0, \ldots\right) . \quad$ If $1 \leq k$, then $\sigma_{k}\left(\Phi_{\iota}^{\lambda}(\pi)\right)=\sigma_{k}^{+}\left(b_{1}\right)$. Hence the assertion is obvious by the definition of $\sigma_{k}^{+}$. Assume that $n+1 \leq k \leq 0$. We see by the definition of $\sigma_{k}^{-}$that

$$
\begin{aligned}
\sigma_{k}^{-}\left(b_{2}\right) & =-\left(q_{k}-p_{k}\right)-\sum_{j=n+1}^{k-1}\left\langle\alpha_{i_{j}}, \alpha_{i_{k}}^{\vee}\right\rangle\left(q_{j}-p_{j}\right) \\
& =-q_{k}+p_{k}-\sum_{j=n+1}^{k-1}\left\langle\alpha_{i_{j}}, \alpha_{i_{k}}^{\vee}\right\rangle q_{j}+\sum_{j=n+1}^{k-1}\left\langle\alpha_{i_{j}}, \alpha_{i_{k}}^{\vee}\right\rangle p_{j} .
\end{aligned}
$$

By Proposition 5.4, we have $\left\langle\operatorname{wt}\left(\Phi_{\iota}^{\lambda}(\pi)\right), \alpha_{i_{k}}^{\vee}\right\rangle=\left\langle x_{n} \lambda, \alpha_{i_{k}}^{\vee}\right\rangle-\sum_{j=n+1}^{m}\left\langle\alpha_{i_{j}}, \alpha_{i_{k}}^{\vee}\right\rangle q_{j}$. Hence,

$$
\begin{aligned}
\sigma_{k}\left(\Phi_{\iota}^{\lambda}(\pi)\right) & =\sigma_{k}^{-}\left(b_{2}\right)-\left\langle\operatorname{wt}\left(\Phi_{\iota}^{\lambda}(\pi)\right), \alpha_{i_{k}}^{\vee}\right\rangle \\
& =-q_{k}+\sum_{j=k}^{m}\left\langle\alpha_{i_{j}}, \alpha_{i_{k}}^{\vee}\right\rangle q_{j}+p_{k}+\sum_{j=n+1}^{k-1}\left\langle\alpha_{i_{j}}, \alpha_{i_{k}}^{\vee}\right\rangle p_{j}-\left\langle x_{n} \lambda, \alpha_{i_{k}}^{\vee}\right\rangle .
\end{aligned}
$$

Because $\left\langle\alpha_{i_{k}}, \alpha_{i_{k}}^{\vee}\right\rangle=2$, we obtain

$$
\begin{aligned}
-q_{k}+\sum_{j=k}^{m}\left\langle\alpha_{i_{j}}, \alpha_{i_{k}}^{\vee}\right\rangle q_{j} & =-q_{k}+\left\langle\alpha_{i_{k}}, \alpha_{i_{k}}^{\vee}\right\rangle q_{k}+\sum_{j=k+1}^{m}\left\langle\alpha_{i_{j}}, \alpha_{i_{k}}^{\vee}\right\rangle q_{j} \\
& =q_{k}+\sum_{j=k+1}^{m}\left\langle\alpha_{i_{j}}, \alpha_{i_{k}}^{\vee}\right\rangle q_{j} .
\end{aligned}
$$

It follows from Lemma 5.3 that $-x_{n} \lambda+x_{k} \lambda+\sum_{j=n+1}^{k} p_{j} \alpha_{i_{j}}=0$, and hence,

$$
\begin{aligned}
0 & =-\left\langle x_{n} \lambda, \alpha_{i_{k}}^{\vee}\right\rangle+\left\langle x_{k} \lambda, \alpha_{i_{k}}^{\vee}\right\rangle+\sum_{j=n+1}^{k}\left\langle\alpha_{i_{j}}, \alpha_{i_{k}}^{\vee}\right\rangle p_{j} \\
& =-\left\langle x_{n} \lambda, \alpha_{i_{k}}^{\vee}\right\rangle+p_{k}+\sum_{j=n+1}^{k-1}\left\langle\alpha_{i_{j}}, \alpha_{i_{k}}^{\vee}\right\rangle p_{j}
\end{aligned}
$$


since $\left\langle x_{k} \lambda, \alpha_{i_{k}}^{\vee}\right\rangle=-p_{k}$ and $\left\langle\alpha_{i_{k}}, \alpha_{i_{k}}^{\vee}\right\rangle=2$. By (5.5) -(5.7), we obtain $\sigma_{k}\left(\Phi_{\iota}^{\lambda}(\pi)\right)=q_{k}+$ $\sum_{j=k+1}^{m}\left\langle\alpha_{i_{j}}, \alpha_{i_{k}}^{\vee}\right\rangle q_{j}$, as desired. If $k \leq n$, then $\sigma_{k}^{-}\left(b_{2}\right)=0$, which implies that $\sigma_{k}\left(\Phi_{\iota}^{\lambda}(\pi)\right)=$ $\sigma_{k}^{-}\left(b_{2}\right)-\left\langle\operatorname{wt}\left(\Phi_{\iota}^{\lambda}(\pi)\right), \alpha_{i_{k}}^{\vee}\right\rangle=-\left\langle\operatorname{wt}\left(\Phi_{\iota}^{\lambda}(\pi)\right), \alpha_{i_{k}}^{\vee}\right\rangle$.

Finally, we assume that $n \leq m \leq 0$. We write $\Phi_{\iota}^{\lambda}(\pi)=(\ldots, 0,0) \otimes t_{\lambda} \otimes b_{2}$ with $b_{2}=\left(-p_{0},-p_{-1}, \ldots,-p_{m+1}, q_{m}-p_{m}, \ldots, q_{n+1}-p_{n+1}, 0, \ldots\right)$, where we understand $b_{2}=$ $\left(q_{0}-p_{0}, q_{-1}-p_{-1}, \ldots, q_{n+1}-p_{n+1}, 0, \ldots\right)$ (resp., $\left.b_{2}=\left(-p_{0},-p_{-1}, \ldots,-p_{n+1}, 0, \ldots\right)\right)$ if $n<m=0$ (resp., $n=m<0$ ). If $1 \leq k$, then it is obvious that $\sigma_{k}\left(\Phi_{\iota}^{\lambda}(\pi)\right)=\sigma_{k}^{+}\left(b_{1}\right)=0$. Assume that $m+1 \leq k \leq 0$. We see that

$$
\begin{aligned}
\sigma_{k}^{-}\left(b_{2}\right) & =-\left(-p_{k}\right)-\sum_{j=m+1}^{k-1}\left\langle\alpha_{i_{j}}, \alpha_{i_{k}}^{\vee}\right\rangle\left(-p_{j}\right)-\sum_{j=n+1}^{m}\left\langle\alpha_{i_{j}}, \alpha_{i_{k}}^{\vee}\right\rangle\left(q_{j}-p_{j}\right) \\
& =p_{k}+\sum_{j=n+1}^{k-1}\left\langle\alpha_{i_{j}}, \alpha_{i_{k}}^{\vee}\right\rangle p_{j}-\sum_{j=n+1}^{m}\left\langle\alpha_{i_{j}}, \alpha_{i_{k}}^{\vee}\right\rangle q_{j} .
\end{aligned}
$$

By Proposition 5.4, we have $\left\langle\operatorname{wt}\left(\Phi_{\iota}^{\lambda}(\pi)\right), \alpha_{i_{k}}^{\vee}\right\rangle=\left\langle x_{n} \lambda, \alpha_{i_{k}}^{\vee}\right\rangle-\sum_{j=n+1}^{m}\left\langle\alpha_{i_{j}}, \alpha_{i_{k}}^{\vee}\right\rangle q_{j}$. Hence,

$$
\sigma_{k}\left(\Phi_{\iota}^{\lambda}(\pi)\right)=\sigma_{k}^{-}\left(b_{2}\right)-\left\langle\operatorname{wt}\left(\Phi_{\iota}^{\lambda}(\pi)\right), \alpha_{i_{k}}^{\vee}\right\rangle=p_{k}+\sum_{j=n+1}^{k-1}\left\langle\alpha_{i_{j}}, \alpha_{i_{k}}^{\vee}\right\rangle p_{j}-\left\langle x_{n} \lambda, \alpha_{i_{k}}^{\vee}\right\rangle .
$$

It follows from Lemma 5.3 that $-x_{n} \lambda+x_{k} \lambda+\sum_{j=n+1}^{k} p_{j} \alpha_{i_{j}}=0$, and hence,

$$
\begin{aligned}
0 & =-\left\langle x_{n} \lambda, \alpha_{i_{k}}^{\vee}\right\rangle+\left\langle x_{k} \lambda, \alpha_{i_{k}}^{\vee}\right\rangle+\sum_{j=n+1}^{k}\left\langle\alpha_{i_{j}}, \alpha_{i_{k}}^{\vee}\right\rangle p_{j} \\
& =-\left\langle x_{n} \lambda, \alpha_{i_{k}}^{\vee}\right\rangle+p_{k}+\sum_{j=n+1}^{k-1}\left\langle\alpha_{i_{j}}, \alpha_{i_{k}}^{\vee}\right\rangle p_{j}
\end{aligned}
$$

since $\left\langle x_{k} \lambda, \alpha_{i_{k}}^{\vee}\right\rangle=-p_{k}$ and $\left\langle\alpha_{i_{k}}, \alpha_{i_{k}}^{\vee}\right\rangle=2$. By (15.8) and (5.9), we obtain $\sigma_{k}\left(\Phi_{\iota}^{\lambda}(\pi)\right)=$ $\sigma_{k}^{-}\left(b_{2}\right)-\left\langle\operatorname{wt}\left(\Phi_{\iota}^{\lambda}(\pi)\right), \alpha_{i_{k}}^{\vee}\right\rangle=0$, as desired. If $k \leq m$, then we can show the equality by the same argument as in the case that $n<0<m$. Thus we have proved the lemma.

Now, we set

$$
i(k):=i_{k}=\left\{\begin{array}{ll}
2 & \text { if } k \text { is even, } \\
1 & \text { if } k \text { is odd },
\end{array} \quad i^{\prime}(k):= \begin{cases}1 & \text { if } k \text { is even } \\
2 & \text { if } k \text { is odd }\end{cases}\right.
$$

for $k \in \mathbb{Z}$; note that

$$
\left\langle x_{k} \lambda, \alpha_{i(k)}^{\vee}\right\rangle=-p_{k}<0, \quad\left\langle x_{k} \lambda, \alpha_{i^{\prime}(k)}^{\vee}\right\rangle=p_{k+1}>0
$$

by (4.4). Let us write $\pi \in \mathbb{B}_{1}(\lambda)$ as (5.1). We see by (5.10) that

$$
H_{i(m)}^{\pi}\left(\frac{q_{m}}{p_{m}}\right)<0=H_{i(m)}^{\pi}(0) .
$$


Moreover, if $m+n$ is odd, then we see that $\left\langle x_{n} \lambda, \alpha_{i(m)}^{\vee}\right\rangle>0$, and hence

$$
H_{i(m)}^{\pi}\left(\frac{q_{n+1}}{p_{n+1}}\right)<\left\langle\operatorname{wt}(\pi), \alpha_{i(m)}^{\vee}\right\rangle=H_{i(m)}^{\pi}(1) .
$$

If $m+n$ is even, then we see that $\left\langle x_{n} \lambda, \alpha_{i^{\prime}(m)}^{\vee}\right\rangle>0$, and hence

$$
H_{i^{\prime}(m)}^{\pi}\left(\frac{q_{n+1}}{p_{n+1}}\right)<\left\langle\operatorname{wt}(\pi), \alpha_{i^{\prime}(m)}^{\vee}\right\rangle=H_{i^{\prime}(m)}^{\pi}(1) .
$$

Lemma 5.6. Let $\pi \in \mathbb{B}_{1}(\lambda)$ be as (5.1). If $n+1 \leq k \leq m$, then

$$
-q_{k}+\sum_{j=k+1}^{m}\left\langle\alpha_{i_{j}}, \alpha_{i_{k}}^{\vee}\right\rangle q_{j}= \begin{cases}H_{i(m)}^{\pi}\left(q_{k} / p_{k}\right) & \text { if } k-m \in 2 \mathbb{Z}, \\ H_{i^{\prime}(m)}^{\pi}\left(q_{k} / p_{k}\right) & \text { if } k-m+1 \in 2 \mathbb{Z} .\end{cases}
$$

Proof. We set $q_{m+1}:=0$ and $q_{n}:=p_{n}$ by convention. Assume that $k-m \in 2 \mathbb{Z}$. Then we obtain

$$
\begin{aligned}
H_{i(m)}^{\pi}\left(\frac{q_{k}}{p_{k}}\right) & =\sum_{j=k}^{m}\left(\frac{q_{j}}{p_{j}}-\frac{q_{j+1}}{p_{j+1}}\right)\left\langle x_{j} \lambda, \alpha_{i(m)}^{\vee}\right\rangle \\
& =\sum_{j=k, k+2, \ldots, m-2}\left(\left(\frac{q_{j}}{p_{j}}-\frac{q_{j+1}}{p_{j+1}}\right)\left(-p_{j}\right)+\left(\frac{q_{j+1}}{p_{j+1}}-\frac{q_{j+2}}{p_{j+2}}\right) p_{j+2}\right)+\frac{q_{m}}{p_{m}}\left(-p_{m}\right) \\
& =\sum_{j=k, k+2, \ldots, m-2}\left(-q_{j}+a_{i(m)} q_{j+1}-q_{j+2}\right)-q_{m} \quad \text { by (4.1) and (4.2) } \\
& =-q_{k}+\sum_{j=k, k+2, \ldots, m-2}\left(a_{i(m)} q_{j+1}-2 q_{j+2}\right) \\
& =-q_{k}+\sum_{j=k+1}^{m}\left\langle\alpha_{i_{j}}, \alpha_{i(m)}^{\vee}\right\rangle q_{j},
\end{aligned}
$$

as desired.

Assume that $k-m+1 \in 2 \mathbb{Z}$. Then we obtain

$$
\begin{aligned}
H_{i^{\prime}(m)}^{\pi}\left(\frac{q_{k}}{p_{k}}\right) & =\sum_{j=k}^{m}\left(\frac{q_{j}}{p_{j}}-\frac{q_{j+1}}{p_{j+1}}\right)\left\langle x_{j} \lambda, \alpha_{i^{\prime}(m)}^{\vee}\right\rangle \\
& =\sum_{j=k, k+2, \ldots, m-1}\left(\left(\frac{q_{j}}{p_{j}}-\frac{q_{j+1}}{p_{j+1}}\right)\left(-p_{j}\right)+\left(\frac{q_{j+1}}{p_{j+1}}-\frac{q_{j+2}}{p_{j+2}}\right) p_{j+2}\right) \\
& =\sum_{j=k, k+2, \ldots, m-1}\left(-q_{j}+a_{i^{\prime}(m)} q_{j+1}-q_{j+2}\right) \quad \text { by (4.1) and (4.2) } \\
& =-q_{k}+\sum_{j=k, k+2, \ldots, m-3}\left(a_{i^{\prime}(m)} q_{j+1}-2 q_{j+2}\right)+a_{i^{\prime}(m)} q_{m}-q_{m+1} \\
& =-q_{k}+\sum_{j=k+1}^{m}\left\langle\alpha_{i_{j}}, \alpha_{i^{\prime}(m)}^{\vee}\right\rangle q_{j},
\end{aligned}
$$

as desired. 
By Lemmas 5.5 and 5.6, we obtain the following proposition.

Proposition 5.7. Let $\pi \in \mathbb{B}_{1}(\lambda)$ be as (5.1). Then,

$$
-\sigma_{k}\left(\Phi_{\iota}^{\lambda}(\pi)\right)= \begin{cases}H_{i(m)}^{\pi}(0) & \text { if } k-m \in 2 \mathbb{Z} \text { and } m+1 \leq k, \\ H_{i(m)}^{\pi}\left(q_{k} / p_{k}\right) & \text { if } k-m \in 2 \mathbb{Z} \text { and } n+1 \leq k \leq m, \\ H_{i(m)}^{\pi}(1) & \text { if } k-m \in 2 \mathbb{Z} \text { and } k \leq n, \\ H_{i^{\prime}(m)}^{\pi}(0) & \text { if } k-m+1 \in 2 \mathbb{Z} \text { and } k \leq m+1, \\ H_{i^{\prime}(m)}^{\pi}\left(q_{k} / p_{k}\right) & \text { if } k-m+1 \in 2 \mathbb{Z} \text { and } n+1 \leq k \leq m, \\ H_{i^{\prime}(m)}^{\pi}(1) & \text { if } k-m+1 \in 2 \mathbb{Z} \text { and } k \leq n .\end{cases}
$$

Proof of Theorem 5.2. By Remark 5.1, it is easy to check that the map $\Phi_{\iota}^{\lambda}$ is injective. We show that $\Phi_{\iota}^{\lambda}$ is a morphism of crystals. Let $\pi \in \mathbb{B}_{1}(\lambda)$. We have $\operatorname{wt}(\pi)=\operatorname{wt}\left(\Phi_{\iota}^{\lambda}(\pi)\right)$ by Proposition 5.4. We show that $\varepsilon_{i}(\pi)=\varepsilon_{i}\left(\Phi_{\iota}^{\lambda}(\pi)\right)$ and $\Phi_{\iota}^{\lambda}\left(\tilde{e}_{i} \pi\right)=\tilde{e}_{i} \Phi_{\iota}^{\lambda}(\pi)$ for $i \in I$. Let us write $\pi$ as (5.1).

Case 1. Assume that $i=i(m)$ and $m+n$ is odd. Note that the function $H_{i}^{\pi}(t)$ attains a minimal value at $t=q_{k} / p_{k}, k=m, m-2, \ldots, n+1$ (see Remark 4.2 and (5.10) ). By (2.7) we have

$$
\begin{aligned}
\varepsilon_{i}(\pi) & =-\min \left\{H_{i}^{\pi}(t) \mid t \in\left\{\frac{q_{m}}{p_{m}}, \frac{q_{m-2}}{p_{m-2}}, \ldots, \frac{q_{n+1}}{p_{n+1}}\right\}\right\} \\
& =\max \left\{-H_{i}^{\pi}(t) \mid t \in\left\{\frac{q_{m}}{p_{m}}, \frac{q_{m-2}}{p_{m-2}}, \ldots, \frac{q_{n+1}}{p_{n+1}}\right\}\right\} .
\end{aligned}
$$

By the definition of $\varepsilon_{i}\left(\Phi_{\iota}^{\lambda}(\pi)\right)$, we have

$$
\varepsilon_{i}\left(\Phi_{\iota}^{\lambda}(\pi)\right)=\max _{k: i_{k}=i} \sigma_{k}\left(\Phi_{\iota}^{\lambda}(\pi)\right)=\max _{k-m \in 2 \mathbb{Z}} \sigma_{k}\left(\Phi_{\iota}^{\lambda}(\pi)\right) .
$$

We see from Proposition 5.7 that

$$
\begin{aligned}
\max _{k-m \in 2 \mathbb{Z}} \sigma_{k}\left(\Phi_{\iota}^{\lambda}(\pi)\right) & =\max \left\{-H_{i}^{\pi}(t) \mid t \in\left\{0, \frac{q_{m}}{p_{m}}, \frac{q_{m-2}}{p_{m-2}}, \ldots, \frac{q_{n+1}}{p_{n+1}}, 1\right\}\right\} \\
& =\max \left\{-H_{i}^{\pi}(t) \mid t \in\left\{\frac{q_{m}}{p_{m}}, \frac{q_{m-2}}{p_{m-2}}, \ldots, \frac{q_{n+1}}{p_{n+1}}\right\}\right\},
\end{aligned}
$$

where the second equality follows from (5.11) and (5.12). By (5.14) $-(\underline{5.16})$, we obtain $\varepsilon_{i}(\pi)=\varepsilon_{i}\left(\Phi_{\iota}^{\lambda}(\pi)\right)$, as desired. Next, we show that $\Phi_{\iota}^{\lambda}\left(\tilde{e}_{i} \pi\right)=\tilde{e}_{i} \Phi_{\iota}^{\lambda}(\pi)$. Since both $\mathbb{B}_{1}(\lambda)$ and $\operatorname{Im}\left(\Psi_{\iota^{+}}^{+}\right) \otimes \mathcal{T}_{\lambda} \otimes \operatorname{Im}\left(\Psi_{\iota^{-}}^{-}\right)$are normal crystals in the sense of [5], the equality $\varepsilon_{i}(\pi)=\varepsilon_{i}\left(\Phi_{\iota}^{\lambda}(\pi)\right)$ and the injectivity of $\Phi_{\iota}^{\lambda}$ imply that

$$
\Phi_{\iota}^{\lambda}\left(\tilde{e}_{i} \pi\right)=\mathbf{0} \Longleftrightarrow \tilde{e}_{i} \pi=\mathbf{0} \Longleftrightarrow \varepsilon_{i}(\pi)=0 \Longleftrightarrow \varepsilon_{i}\left(\Phi_{\iota}^{\lambda}(\pi)\right)=0 \Longleftrightarrow \tilde{e}_{i} \Phi_{\iota}^{\lambda}(\pi)=\mathbf{0} .
$$

Assume that $\tilde{e}_{i} \pi \neq \mathbf{0}$, or equivalently, $\tilde{e}_{i} \Phi_{\iota}^{\lambda}(\pi) \neq \mathbf{0}$. By the definition of $\Phi_{\iota}^{\lambda}$, we have $\Phi_{\iota}^{\lambda}(\pi)=\left(\ldots, y_{2}, y_{1}\right) \otimes t_{\lambda} \otimes\left(y_{0}, y_{-1}, \ldots\right)$, where $y_{k}=z_{k}(\pi)$ (see (15.2)). Let $M_{(i)}$ be as 
(2.8), and set $k^{\prime}:=\max M_{(i)}$. Namely, $k^{\prime}$ is the largest integer $k$ such that $\sigma_{(i)}\left(\Phi_{\iota}^{\lambda}(\pi)\right)=$ $\sigma_{k}\left(\Phi_{\iota}^{\lambda}(\pi)\right)$ and $k-m \in 2 \mathbb{Z}$. Then we see by the definition of $\tilde{e}_{i}$ that $\tilde{e}_{i} \Phi_{\iota}^{\lambda}(\pi)=\left(\ldots, y_{2}^{\prime}, y_{1}^{\prime}\right) \otimes$ $t_{\lambda} \otimes\left(y_{0}^{\prime}, y_{-1}^{\prime}, \ldots\right)$, where $y_{k}^{\prime}:=y_{k}-\delta_{k, k^{\prime}}$. Let $t_{1}$ and $t_{0}$ be as (2.4) and (2.5)), respectively. By (5.14) - (5.16), we obtain $t_{1}=q_{k^{\prime}} / p_{k^{\prime}}$. By (2.6) and Remark 4.2, we have $t_{0}=t_{1}-$ $1 /\left(-\left\langle x_{k^{\prime}} \lambda, \alpha_{i}^{\vee}\right\rangle\right)=\left(q_{k^{\prime}}-1\right) / p_{k^{\prime}}$. Assume that $k^{\prime}<m$. By (2.6) and Remark 4.2, we have $q_{k^{\prime}+1} / p_{k^{\prime}+1} \leq t_{0}$. Suppose, for a contradiction, that $q_{k^{\prime}+1} / p_{k^{\prime}+1}=t_{0}$, that is,

$$
H_{i}^{\pi}\left(\frac{q_{k^{\prime}+1}}{p_{k^{\prime}+1}}\right)=H_{i}^{\pi}\left(\frac{q_{k^{\prime}}}{p_{k^{\prime}}}\right)+1
$$

Then it follows from Remark 4.2 that $H_{i}^{\pi}(t)$ attains a minimal value at $t=q_{k^{\prime}+2} / p_{k^{\prime}+2}$, and hence $H_{i}^{\pi}\left(q_{k^{\prime}+2} / p_{k^{\prime}+2}\right) \in \mathbb{Z}$ by (2.3). By (5.17), we obtain $H_{i}^{\pi}\left(q_{k^{\prime}+2} / p_{k^{\prime}+2}\right) \leq H_{i}^{\pi}\left(q_{k^{\prime}} / p_{k^{\prime}}\right)$, which contradicts the definition of $t_{1}$. Therefore we obtain $q_{k^{\prime}+1} / p_{k^{\prime}+1}<t_{0}$ and

$$
\tilde{e}_{i} \pi=\left(x_{m} \lambda, \ldots, x_{k^{\prime}} \lambda \ldots, x_{n} \lambda ; 0, \frac{q_{m}}{p_{m}} \ldots, \frac{q_{k^{\prime}+1}}{p_{k^{\prime}+1}}, \frac{q_{k^{\prime}}-1}{q_{k^{\prime}}}, \frac{q_{k^{\prime}-1}}{p_{k^{\prime}-1}}, \ldots, \frac{q_{n+1}}{p_{n+1}}, 1\right) .
$$

If $k^{\prime}=m$, then

$$
\tilde{e}_{i} \pi= \begin{cases}\left(x_{m} \lambda, \ldots, x_{n} \lambda ; 0, \frac{q_{m}-1}{q_{m}}, \frac{q_{m-1}}{p_{m-1}}, \ldots, \frac{q_{n+1}}{p_{n+1}}, 1\right) & \text { if } q_{m}>1, \\ \left(x_{m-1} \lambda, \ldots, x_{n} \lambda ; 0, \frac{q_{m-1}}{p_{m-1}}, \ldots, \frac{q_{n+1}}{p_{n+1}}, 1\right) & \text { if } q_{m}=1 .\end{cases}
$$

Hence we see that

$$
\begin{aligned}
& z_{k}\left(\tilde{e}_{i} \pi\right)= \begin{cases}q_{k^{\prime}}-1 & \text { if } 1 \leq k=k^{\prime}, \\
\left(q_{k^{\prime}}-1\right)-p_{k^{\prime}} & \text { if } k=k^{\prime} \leq 0, \\
q_{k} & \text { if } k \neq k^{\prime}, 1 \leq k, \text { and } n+1 \leq k \leq m, \\
p_{k} & \text { if } k \neq k^{\prime}, 1 \leq k, \text { and } k \leq n, \\
q_{k}-p_{k} & \text { if } k \neq k^{\prime}, k \leq 0, \text { and } n+1 \leq k \leq m, \\
-p_{k} & \text { if } k \neq k^{\prime}, k \leq 0, \text { and } m+1 \leq k, \\
0 & \text { otherwise, }\end{cases} \\
&=z_{k}(\pi)-\delta_{k, k^{\prime}},
\end{aligned}
$$

which implies that $\Phi_{\iota}^{\lambda}\left(\tilde{e}_{i} \pi\right)=\tilde{e}_{i} \Phi_{\iota}^{\lambda}(\pi)$.

Case 2. Assume that $i=i^{\prime}(m)$ and $m+n$ is even. Note that the function $H_{i}^{\pi}(t)$ attains a minimal value at $t=0$ and $t=q_{k} / p_{k}, k=m-1, m-3, \ldots, n+1$. As in Case 1 , we deduce by Proposition 5.7 and (5.13) that

$$
\begin{aligned}
\varepsilon_{i}(\pi) & =-\min \left\{H_{i}^{\pi}(t) \mid t \in\left\{0, \frac{q_{m-1}}{p_{m-1}}, \frac{q_{m-3}}{p_{m-3}}, \ldots, \frac{q_{n+1}}{p_{n+1}}\right\}\right\} \\
& =\max \left\{-H_{i}^{\pi}(t) \mid t \in\left\{0, \frac{q_{m-1}}{p_{m-1}}, \frac{q_{m-3}}{p_{m-3}}, \ldots, \frac{q_{n+1}}{p_{n+1}}\right\}\right\} \\
& =\max _{k-m+1 \in 2 \mathbb{Z}} \sigma_{k}\left(\Phi_{\iota}^{\lambda}(\pi)\right)=\varepsilon_{i}\left(\Phi_{\iota}^{\lambda}(\pi)\right) .
\end{aligned}
$$


We can show that $\Phi_{\iota}^{\lambda}\left(\tilde{e}_{i} \pi\right)=\tilde{e}_{i} \Phi_{\iota}^{\lambda}(\pi)$ in exactly the same way as Case 1 .

Case 3. Assume that $i=i(m)$ and $m+n$ is even. Note that the function $H_{i}^{\pi}(t)$ attains a minimal value at $t=q_{k} / p_{k}, k=m, m-2, \ldots, n+2$ and $t=1$. As in Case 1 , we deduce by Proposition 5.7 and (5.11) that

$$
\begin{aligned}
\varepsilon_{i}(\pi) & =-\min \left\{H_{i}^{\pi}(t) \mid t \in\left\{\frac{q_{m}}{p_{m}}, \frac{q_{m-2}}{p_{m-2}}, \ldots, \frac{q_{n+2}}{p_{n+2}}, 1\right\}\right\} \\
& =\max \left\{-H_{i}^{\pi}(t) \mid t \in\left\{\frac{q_{m}}{p_{m}}, \frac{q_{m-2}}{p_{m-2}}, \ldots, \frac{q_{n+2}}{p_{n+2}}, 1\right\}\right\} \\
& =\max _{k-m \in 2 \mathbb{Z}} \sigma_{k}\left(\Phi_{\iota}^{\lambda}(\pi)\right)=\varepsilon_{i}\left(\Phi_{\iota}^{\lambda}(\pi)\right) .
\end{aligned}
$$

We show that $\Phi_{\iota}^{\lambda}\left(\tilde{e}_{i} \pi\right)=\tilde{e}_{i} \Phi_{\iota}^{\lambda}(\pi)$. If $m=n$, then $\pi=\left(x_{n} \lambda ; 0,1\right)$. We see by definition of $\Phi_{\iota}^{\lambda}$ that

$$
\Phi_{\iota}^{\lambda}(\pi)= \begin{cases}\left(\ldots, 0, p_{n}, \ldots, p_{2}, p_{1}\right) \otimes t_{\lambda} \otimes(0,0, \ldots) & \text { if } n>0 \\ (\ldots, 0,0) \otimes t_{\lambda} \otimes(0,0, \ldots) & \text { if } n=0 \\ (\ldots, 0,0) \otimes t_{\lambda} \otimes\left(-p_{0},-p_{-1}, \ldots,-p_{n+1}, 0, \ldots\right) & \text { if } n<0\end{cases}
$$

Also, we see that

$$
\tilde{e}_{i} \pi= \begin{cases}\left(x_{n} \lambda, x_{n-1} \lambda ; 0,\left(p_{n}-1\right) / p_{n}, 1\right) & \text { if } p_{n}>1 \\ \left(x_{n-1} \lambda ; 0,1\right) & \text { if } p_{n}=1\end{cases}
$$

Thus it is easy to verify that $\Phi_{\iota}^{\lambda}\left(\tilde{e}_{i} \pi\right)=\tilde{e}_{i} \Phi_{\iota}^{\lambda}(\pi)$ in this case. Assume that $m>n$; by the assumption that $m+n$ is even, we have $m \geq n+2$. Let $M_{(i)}$ be as (2.8), and set $k^{\prime}:=\max M_{(i)}$. If $k^{\prime} \in\{m, m-2, \ldots, n+2\}$, then we can show in exactly the same way as Case 1 that $\Phi_{\iota}^{\lambda}\left(\tilde{e}_{i}(\pi)\right)=\tilde{e}_{i} \Phi_{\iota}^{\lambda}(\pi)$. Otherwise, we see by Proposition 5.7 and (5.18) that $k^{\prime}=n$. Let $\Phi_{\iota}^{\lambda}(\pi)=\left(\ldots, y_{2}, y_{1}\right) \otimes t_{\lambda} \otimes\left(y_{0}, y_{-1}, \ldots\right)$, where $y_{k}=z_{k}(\pi)$. Then we see by the definition of $\tilde{e}_{i}$ that $\tilde{e}_{i} \Phi_{\iota}^{\lambda}(\pi)=\left(\ldots, y_{2}^{\prime}, y_{1}^{\prime}\right) \otimes t_{\lambda} \otimes\left(y_{0}^{\prime}, y_{-1}^{\prime}, \ldots\right)$, where $y_{k}^{\prime}=y_{k}-\delta_{k, n}$. Let $t_{1}$ and $t_{0}$ be as (2.4) and (2.5), respectively. We see that $t_{1}=1$ and $t_{0}=1-1 /\left(-\left\langle x_{n} \lambda, \alpha_{i}^{\vee}\right\rangle\right)=1-1 / p_{n-1}$. By (2.6) and Remark 4.2, we have $q_{n+1} / p_{n+1} \leq t_{0}$. Suppose, for a contradiction, that $q_{n+1} / p_{n+1}=t_{0}$; note that $H_{i}^{\pi}\left(q_{n+1} / p_{n+1}\right)=H_{i}^{\pi}(1)+1$. It follows from Remark 4.2 that $H_{i}^{\pi}(t)$ attains a minimal value at $t=q_{n+2} / p_{n+2}$, and hence $H_{i}^{\pi}\left(q_{n+2} / p_{n+2}\right) \in \mathbb{Z}$ by (2.3) . Therefore, we obtain $H_{i}^{\pi}\left(q_{n+2} / p_{n+2}\right) \leq H_{i}^{\pi}(1)$, which contradicts the definition of $t_{1}$. Therefore we obtain $q_{n+1} / p_{n+1}<t_{0}$, which implies that

$$
\tilde{e}_{i} \pi=\left(x_{m} \lambda, \ldots, x_{n} \lambda, x_{n-1} \lambda ; 0, \frac{q_{m}}{p_{m}} \ldots, \ldots, \frac{q_{n+1}}{p_{n+1}}, \frac{p_{n}-1}{p_{n}}, 1\right) .
$$


Therefore we see that

$$
\begin{aligned}
& z_{k}\left(\tilde{e}_{i} \pi\right)= \begin{cases}p_{n}-1 & \text { if } 1 \leq k=n \\
\left(p_{n}-1\right)-p_{n} & \text { if } k=n \leq 0, \\
q_{k} & \text { if } k \neq n, 1 \leq k, \text { and } n+1 \leq k \leq m, \\
p_{k} & \text { if } k \neq n, 1 \leq k, \text { and } k \leq n, \\
q_{k}-p_{k} & \text { if } k \neq n, k \leq 0, \text { and } n+1 \leq k \leq m, \\
-p_{k} & \text { if } k \neq n, k \leq 0, \text { and } m+1 \leq k, \\
0 & \text { otherwise }\end{cases} \\
&=z_{k}(\pi)-\delta_{k, n} .
\end{aligned}
$$

Hence we obtain $\tilde{e}_{i}(\pi)=\tilde{e}_{i} \Phi_{\iota}^{\lambda}(\pi)$, as desired.

Case 4. Assume that $i=i^{\prime}(m)$ and $m+n$ is odd. Note that the function $H_{i}^{\pi}(t)$ attains a minimal value at $t=0, t=1$, and $t=q_{k} / p_{k}, k=m-1, m-3, \ldots, n+2$. By Proposition 5.7, we get

$$
\begin{aligned}
\varepsilon_{i}(\pi) & =-\min \left\{H_{i}^{\pi}(t) \mid t \in\left\{0, \frac{q_{m-1}}{p_{m-1}}, \frac{q_{m-3}}{p_{m-3}}, \ldots, \frac{q_{n+2}}{p_{n+2}}, 1\right\}\right\} \\
& =\max \left\{-H_{i}^{\pi}(t) \mid t \in\left\{0, \frac{q_{m-1}}{p_{m-1}}, \frac{q_{m-3}}{p_{m-3}}, \ldots, \frac{q_{n+2}}{p_{n+2}}, 1\right\}\right\} \\
& =\varepsilon_{i}\left(\Phi_{\iota}^{\lambda}(\pi)\right) .
\end{aligned}
$$

We can show in exactly the same way as Case 3 that $\Phi_{\iota}^{\lambda}\left(\tilde{e}_{i} \pi\right)=\tilde{e}_{i} \Phi_{\iota}^{\lambda}(\pi)$.

Let $\pi \in \mathbb{B}_{1}(\lambda)$, and $i \in I$. Because $\operatorname{wt}(\pi)=\operatorname{wt}\left(\Phi_{\iota}^{\lambda}(\pi)\right)$ and $\varepsilon_{i}(\pi)=\varepsilon_{i}\left(\Phi_{\iota}^{\lambda}(\pi)\right)$, we have $\varphi_{i}(\pi)=\varphi_{i}\left(\Phi_{\iota}^{\lambda}(\pi)\right)$. Also, since both $\mathbb{B}_{1}(\lambda)$ and $\operatorname{Im}\left(\Psi_{\iota^{+}}^{+}\right) \otimes \mathcal{T}_{\lambda} \otimes \operatorname{Im}\left(\Psi_{\iota^{-}}^{-}\right)$are normal crystals, and since $\Phi_{\iota}^{\lambda}\left(\tilde{e}_{i} \pi\right)=\tilde{e}_{i} \Phi_{\iota}^{\lambda}(\pi)$, we see that $\Phi_{\iota}^{\lambda}\left(\tilde{f}_{i} \pi\right)=\tilde{f}_{i} \Phi_{\iota}^{\lambda}(\pi)$. This completes the proof of Theorem 5.2 .

\subsection{Proof of Theorem 3.3.}

In this subsection, we assume that $\lambda \in P$ is of the form either $k_{1} \Lambda_{1}-\Lambda_{2}$ with $1 \leq$ $k_{1}<a_{1}-1$ or $\Lambda_{1}-k_{2} \Lambda_{2}$ with $1<k_{2} \leq a_{2}-1$; note that $\lambda$ satisfies the condition that $W \lambda \cap\left(P^{+} \cup-P^{+}\right)=\emptyset($ see $\$ 3)$.

We can prove Theorem 3.3 in exactly the same way as [14, Theorem 3.2]. So, we give only a sketch of the proof. In the following, we assume that $\lambda=k_{1} \Lambda_{1}-\Lambda_{2}$ with $1 \leq k_{1}<a_{1}-1$; the proof for the case that $\lambda=\Lambda_{1}-k_{2} \Lambda_{2}$ with $1<k_{2} \leq a_{2}-1$ is similar. Let us identify $\mathcal{B}(\lambda)$ with $\left\{b \in \mathcal{B}(\infty) \otimes \mathcal{T}_{\mu} \otimes \mathcal{B}(-\infty) \mid b^{*}\right.$ is extremal $\}$ by Theorem 2.4 .

Lemma 5.8 (cf. [14, Lemmas 3.7 and 3.8]). (1) Let $i \in I$ and $b \in \mathcal{B}(\lambda)$ be such that $\tilde{e}_{i} b \neq \mathbf{0}$. If $b$ is of the form $b=b_{1} \otimes t_{\lambda} \otimes u_{-\infty}$ with $b_{1} \neq u_{\infty}$, then $\tilde{e}_{i} b=\tilde{e}_{i} b_{1} \otimes t_{\lambda} \otimes u_{-\infty}$. 
(2) Let $i \in I$ and $b \in \mathcal{B}(\lambda)$ be such that $\tilde{f}_{i} b \neq \mathbf{0}$. If $b$ is of the form $b=u_{\infty} \otimes t_{\lambda} \otimes b_{2}$ with $b_{2} \neq u_{-\infty}$, then $\tilde{f}_{i} b=u_{\infty} \otimes t_{\lambda} \otimes \tilde{f}_{i} b_{2}$.

Proof. We give a proof only for part (11). Suppose, for a contradiction, that $\tilde{e}_{i} b=b_{1} \otimes$ $t_{\lambda} \otimes \tilde{e}_{i} u_{-\infty}$. We see by $(2.2)$ that $\left(\tilde{e}_{i} b\right)^{*}=b_{1}^{*} \otimes t_{-\lambda-\mathrm{wt}\left(b_{1}\right)-\alpha_{i}} \otimes \tilde{e}_{i} u_{-\infty}$. Since $\varphi_{i}\left(\left(\tilde{e}_{i} b\right)^{*}\right) \geq$ $\varphi_{i}\left(\tilde{e}_{i} u_{-\infty}\right)=1$, it follows from the tensor product rule of crystals that $\tilde{f}_{i}\left(\tilde{e}_{i} b\right)^{*} \neq \mathbf{0}$. Because $\tilde{e}_{i} b \in \mathcal{B}(\lambda)$, we see that $\left(\tilde{e}_{i} b\right)^{*}$ is an extremal element of weight $-\lambda$. Since $\left\langle\operatorname{wt}\left(S_{\text {id }}\left(\tilde{e}_{i} b\right)^{*}\right), \alpha_{1}^{\vee}\right\rangle=\left\langle-\lambda, \alpha_{1}^{\vee}\right\rangle=-k_{1} \leq 0$, we obtain $\tilde{f}_{1}\left(\tilde{e}_{i} b\right)^{*}=\mathbf{0}$. Therefore we have $i=2$ and $\left(\tilde{e}_{2} b\right)^{*}=b_{1}^{*} \otimes t_{-\lambda-\operatorname{wt}\left(b_{1}\right)-\alpha_{2}} \otimes \tilde{e}_{2} u_{-\infty}$. Because $\left\langle\operatorname{wt}\left(S_{\text {id }}\left(\tilde{e}_{2} b\right)^{*}\right), \alpha_{2}^{\vee}\right\rangle=\left\langle-\lambda, \alpha_{2}^{\vee}\right\rangle=$ $1 \geq 0$, and $\left(\tilde{e}_{2} b\right)^{*}$ is an extremal element of extremal weight $-\lambda$, we see that $\tilde{e}_{2}\left(\tilde{e}_{2} b\right)^{*}=\mathbf{0}$, and hence $\varepsilon_{2}\left(\left(\tilde{e}_{2} b\right)^{*}\right)=0$. Since $\varepsilon_{2}\left(\left(\tilde{e}_{2} b\right)^{*}\right) \geq \varepsilon_{2}\left(b_{1}^{*}\right)$, we have $\varepsilon_{2}\left(b_{1}^{*}\right)=0$, which implies $\varepsilon_{1}\left(b_{1}^{*}\right) \geq 1$ because $b_{1} \neq u_{\infty}$. Hence

$$
\begin{aligned}
\varphi_{2}\left(b_{1}^{*} \otimes t_{-\lambda-\operatorname{wt}\left(b_{1}\right)-\alpha_{2}}\right) & =\varphi_{2}\left(b_{1}^{*}\right)+\left\langle-\lambda-\operatorname{wt}\left(b_{1}\right)-\alpha_{2}, \alpha_{2}^{\vee}\right\rangle \\
& =\left(\varepsilon_{2}\left(b_{1}^{*}\right)+\left\langle\operatorname{wt}\left(b_{1}^{*}\right), \alpha_{2}^{\vee}\right\rangle\right)+\left\langle-\lambda-\operatorname{wt}\left(b_{1}\right)-\alpha_{2}, \alpha_{2}^{\vee}\right\rangle \\
& =\varepsilon_{2}\left(b_{1}^{*}\right)+\left\langle-\lambda-\alpha_{2}, \alpha_{2}^{\vee}\right\rangle=-1 .
\end{aligned}
$$

By this equality and $\varepsilon_{2}\left(\tilde{e}_{2} u_{-\infty}\right)=\varphi_{2}\left(\tilde{e}_{2} u_{-\infty}\right)-\left\langle\operatorname{wt}\left(\tilde{e}_{2} u_{-\infty}\right), \alpha_{2}^{\vee}\right\rangle=-1$, it follows from the tensor product rule of crystals that $S_{2}\left(\tilde{e}_{2} b\right)^{*}=\tilde{f}_{2}\left(\tilde{e}_{2} b\right)^{*}=b_{1}^{*} \otimes t_{-\lambda-\operatorname{wt}\left(b_{1}\right)-\alpha_{2}} \otimes u_{-\infty}$. Since $\varepsilon_{1}\left(b_{1}^{*}\right) \geq 1$, we obtain $\tilde{e}_{1} b_{1}^{*} \neq \mathbf{0}$. Therefore it follows from the tensor product rule of crystals that $\varepsilon_{1}\left(S_{2}\left(\tilde{e}_{2} b\right)^{*}\right) \geq \varepsilon_{1}\left(b_{1}^{*}\right) \geq 1$, that is, $\tilde{e}_{1} S_{2}\left(\tilde{e}_{2} b\right)^{*} \neq \mathbf{0}$. However, since $\left(\tilde{e}_{2} b\right)^{*}$ is an extremal element of weight $-\lambda$ and $\left\langle\operatorname{wt}\left(S_{2}\left(\tilde{e}_{2} b\right)^{*}\right), \alpha_{1}^{\vee}\right\rangle=\left\langle s_{2}(-\lambda), \alpha_{1}^{\vee}\right\rangle \geq 0$, we see that $\tilde{e}_{1} S_{2}\left(\tilde{e}_{2} b\right)^{*}=\mathbf{0}$, which is a contradiction.

Lemma 5.8 implies the following proposition (see [14, Proposition 3.9]).

Proposition 5.9. It holds that $\mathcal{B}(\lambda) \subset\left(\mathcal{B}(\infty) \otimes t_{\lambda} \otimes u_{-\infty}\right) \cup\left(u_{\infty} \otimes t_{\lambda} \otimes \mathcal{B}(-\infty)\right)$.

Here, we set $|\alpha|:=\sum_{i \in I}\left|c_{i}\right|$ for $\alpha=\sum_{i \in I} c_{i} \alpha_{i} \in \bigoplus_{i \in I} \mathbb{Z} \alpha_{i}$. By Proposition [5.9, we see that $b \in \mathcal{B}(\lambda)$ is of the form either $b=b_{1} \otimes t_{\lambda} \otimes u_{-\infty}$ with some $b_{1} \in \mathcal{B}(\infty)$ or $b=u_{\infty} \otimes t_{\lambda} \otimes b_{2}$ with some $b_{2} \in \mathcal{B}(-\infty)$. We deduce by induction on $\left|\operatorname{wt}\left(b_{1}\right)\right|\left(\operatorname{resp} .,\left|\operatorname{wt}\left(b_{2}\right)\right|\right)$ that if $b$ is of the form $b_{1} \otimes t_{\lambda} \otimes u_{-\infty}$ (resp., $\left.b=u_{\infty} \otimes t_{\lambda} \otimes b_{2}\right)$, then $b=\tilde{f}_{i_{r}} \cdots \tilde{f}_{i_{1}} u_{\lambda}$ (resp., $b=\tilde{e}_{i_{r}} \cdots \tilde{e}_{i_{1}} u_{\lambda}$ ) for some $i_{1}, \ldots, i_{r}$ (see [14, Proof of Theorem 3.2]). Thus we have proved Theorem 3.3 .

Remark 5.10. Set

$$
\begin{aligned}
& \mathcal{B}(\lambda)_{-}:=\left\{\tilde{f}_{i_{l}} \cdots \tilde{f}_{i_{2}} \tilde{f}_{i_{1}} u_{\lambda} \mid i_{1}, i_{2}, \ldots i_{l} \in I, l \geq 1\right\} \backslash\{\mathbf{0}\}, \\
& \mathcal{B}(\lambda)_{+}:=\left\{\tilde{e}_{i_{l}} \cdots \tilde{e}_{i_{2}} \tilde{e}_{i_{1}} u_{\lambda} \mid i_{1}, i_{2}, \ldots i_{l} \in I, l \geq 1\right\} \backslash\{\mathbf{0}\} .
\end{aligned}
$$

By Theorem $[3.3$, we can decompose $\mathcal{B}(\lambda)$ as

$$
\mathcal{B}(\lambda)=\mathcal{B}(\lambda)_{-} \sqcup\left\{u_{\lambda}\right\} \sqcup \mathcal{B}(\lambda)_{+} \cdot
$$

In particular, we see that $\# \mathcal{B}(\lambda)_{\mu}<\infty$, where $\mathcal{B}(\lambda)_{\mu}:=\{b \in \mathcal{B}(\lambda) \mid \operatorname{wt}(b)=\mu\}$. 


\section{Computation of $\# \mathcal{B}\left(\Lambda_{1}-\Lambda_{2}\right)_{\mu}$.}

In this section, we assume that

$$
A=\left(\begin{array}{cc}
2 & -a \\
-a & 2
\end{array}\right) \quad \text { with } a \geq 3, \text { and } \lambda=\Lambda_{1}-\Lambda_{2} \text {. }
$$

By Corollary 3.4 (see also [14, Theorem 3.6]), we have $\mathcal{B}(\lambda) \cong \mathbb{B}(\lambda)$. The aim of this section is to give an algorithm for computing $\operatorname{dim} V(\lambda)_{\mu}=\# \mathcal{B}(\lambda)_{\mu}$ for $\mu \in P$.

\subsection{Subsets of $\mathbb{Z}_{\geq 0}^{+\infty}$ and $\mathbb{Z}_{\leq 0}^{-\infty}$.}

We define maps $F$ and $F^{\prime}$ as follows. Recall that the sequence $\left\{p_{m}\right\}$ is defined by recursive formulas (4.1) and (4.2) for $\lambda=\Lambda_{1}-\Lambda_{2}$; we know from [1, Lemma 3.5] that

$$
\cdots p_{3}>p_{2}>p_{1}=1=p_{0}=1<p_{-1}<p_{-2}<\cdots .
$$

For $x \in \mathbb{Z}_{\geq 2}$, we denote by $n(x)$ the (unique) positive integer such that $p_{n(x)-1}<x \leq p_{n(x)}$, and set $n(1):=1$. For $x \in \mathbb{Z}_{\geq 1}$, we define $F(x)$ to be the (unique) integer such that

$$
\frac{F(x)}{p_{n(x)+1}} \leq \frac{x}{p_{n(x)}}<\frac{F(x)+1}{p_{n(x)+1}}
$$

and set $F(0):=0$; note that

$$
n\left(p_{m}\right)=m \text { and } F\left(p_{m}\right)=p_{m+1} \quad \text { for some } m \geq 1 .
$$

Similarly, for $x \in \mathbb{Z}_{\leq-2}$, we denote by $n^{\prime}(x)$ the negative integer such that $-p_{n^{\prime}(x)} \leq x<$ $-p_{n^{\prime}(x)+1}$, and set $n^{\prime}(-1):=0$. For $x \in \mathbb{Z}_{\leq-1}$, we define $F^{\prime}(x)$ to be the integer such that

$$
\frac{F^{\prime}(x)-1}{p_{n^{\prime}(x)-1}}<\frac{x}{p_{n^{\prime}(x)}} \leq \frac{F^{\prime}(x)}{p_{n^{\prime}(x)-1}},
$$

and set $F^{\prime}(0):=0$; note that

$$
n^{\prime}\left(-p_{m}\right)=m \text { and } F^{\prime}\left(-p_{m}\right)=-p_{m-1} \quad \text { for some } m \leq 0 .
$$

Lemma 6.1. (1) Let $x \in \mathbb{Z}_{\geq 1}$. For all $m \geq n(x)$, we have

$$
\frac{F(x)}{p_{m+1}} \leq \frac{x}{p_{m}}<\frac{F(x)+1}{p_{m+1}} .
$$

(2) Let $x \in \mathbb{Z}_{\leq-1}$. For all $m \leq n^{\prime}(x)$, we have

$$
\frac{F^{\prime}(x)-1}{p_{m-1}}<\frac{x}{p_{m}} \leq \frac{F^{\prime}(x)}{p_{m-1}} .
$$


Proof. We give a proof only for part (11); the proof for part (2) is similar. We show the following inequalities, which are equivalent to (6.3):

$$
\frac{F(x)}{x} \leq \frac{p_{m+1}}{p_{m}}<\frac{F(x)+1}{x} .
$$

Because the sequence $\left\{p_{m+1} / p_{m}\right\}_{m \geq 1}$ is an increasing sequence, the first inequality is obvious by the definition (6.1) of $F(x)$. We show the second inequality. Suppose, for a contradiction, that there exists $m \geq n(x)$ such that

$$
\frac{p_{m+1}}{p_{m}}<\frac{F(x)+1}{x} \leq \frac{p_{m+2}}{p_{m+1}} .
$$

We compute

$$
\begin{aligned}
p_{m}(F(x)+1)-x p_{m+1} & \leq p_{m}\left(\frac{p_{m+2}}{p_{m+1}} x\right)-x p_{m+1} \quad \text { by the second inequality in (6.4) } \\
& =\frac{x}{p_{m+1}}\left(p_{m} p_{m+2}-p_{m+1}^{2}\right) \\
& =\frac{x}{p_{m+1}}\left(p_{0} p_{2}-p_{1}^{2}\right) \quad \text { by (4.1) } \\
& =\frac{x}{p_{m+1}}\left(1(a-1)-1^{2}\right) \\
& \leq \frac{p_{n(x)}}{p_{n(x)+1}}(a-2) \quad \text { by } n(x) \leq m \text { and } x \leq p_{n(x)} \\
& \leq \frac{p_{1}}{p_{2}}(a-2) \quad \text { since }\left\{p_{m} / p_{m+1}\right\}_{m \geq 1} \text { is decreasing } \\
& =\frac{1}{a-1}(a-2)<1 \quad \text { since } a \geq 3 .
\end{aligned}
$$

However, by the first inequality in (6.4), we obtain $p_{m}(F(x)+1)-x p_{m+1}>0$, which contradicts the fact that $p_{m}(F(x)+1)-x p_{m+1}$ is a integer. Thus we have proved (6.3) .

The next corollary follows from Lemma 6.1 and the facts that

$$
\lim _{m \rightarrow+\infty} \frac{p_{m+1}}{p_{m}}=\frac{a+\sqrt{a^{2}-4}}{2} \text { and } \lim _{m \rightarrow-\infty} \frac{p_{m-1}}{p_{m}}=\frac{a+\sqrt{a^{2}-4}}{2} .
$$

Corollary 6.2. For $x \in \mathbb{Z}_{\geq 0}$,

$$
F(x)=\left\lfloor\frac{a+\sqrt{a^{2}-4}}{2} x\right\rfloor,
$$

and for $x \in \mathbb{Z}_{\leq 0}$,

$$
F^{\prime}(x)=\left\lceil\frac{a+\sqrt{a^{2}-4}}{2} x\right\rceil
$$


Now, we set

$$
\begin{aligned}
& Y_{-}:=\left\{\left(\ldots, y_{j}, \ldots, y_{2}, y_{1}\right) \in \mathbb{Z}_{\geq 0}^{+\infty} \mid y_{j+1} \leq F\left(y_{j}\right) \text { for all } j \geq 1\right\} \\
& Y_{+}:=\left\{\left(y_{0}, y_{-1}, \ldots, y_{j}, \ldots,\right) \in \mathbb{Z}_{\leq 0}^{-\infty} \mid F^{\prime}\left(y_{j}\right) \leq y_{j-1} \text { for all } j \leq 0\right\}
\end{aligned}
$$

Remark 6.3. Let $y=\left(\ldots, y_{j}, \ldots y_{2}, y_{1}\right) \in Y_{-}$. If there exists $l \in \mathbb{Z}_{\geq 1}$ such that $y_{l}=$ 0 , then we see by $F(0)=0$ that $y_{j}=0$ for all $j>l$. Therefore, if $y \neq(\ldots 0,0)$, then $y$ is of the form $y=\left(\ldots, 0, y_{m}, \ldots y_{2}, y_{1}\right)$ for some $m \in \mathbb{Z}_{\geq 1}$, where $y_{j}>0$ for all $1 \leq j \leq m$. Similarly, we see that $y \in Y_{+}$is of the form either $y=(0,0, \ldots)$ or $y=\left(y_{0}, y_{-1}, \ldots, y_{m}, 0, \ldots,\right)$ for some $m \in \mathbb{Z}_{\leq 0}$, where $y_{j}<0$ for all $m \leq j \leq 0$.

For $n_{1}, n_{2} \geq 0$ and $0 \leq m \leq n_{1}$, we set

$$
Y_{-}\left(n_{1}, n_{2} ; m\right):=\left\{\left(\ldots, y_{j}, \ldots, y_{2}, y_{1}\right) \in Y_{-} \mid y_{1}=m, \sum_{j: \text { odd }} y_{j}=n_{1}, \sum_{j: \text { even }} y_{j}=n_{2}\right\}
$$

and for $n_{1}, n_{2} \leq 0$ and $n_{2} \leq m \leq 0$, we set

$$
Y_{+}\left(n_{1}, n_{2} ; m\right):=\left\{\left(y_{0}, y_{-1}, \ldots, y_{j}, \ldots\right) \in Y_{+} \mid y_{0}=m, \sum_{j: \text { odd }} y_{j}=n_{1}, \sum_{j: \text { even }} y_{j}=n_{2}\right\} .
$$

We give an algorithm for computing $\# Y_{-}\left(n_{1}, n_{2} ; m\right)$. It is obvious by Remark 6.3 that for $n_{1}, n_{2} \geq 0$,

$$
\# Y_{-}\left(n_{1}, n_{2} ; 0\right)= \begin{cases}1 & \text { if } n_{1}=n_{2}=0 \\ 0 & \text { otherwise }\end{cases}
$$

Assume that $1 \leq m \leq n_{1}$. We have $Y_{-}(m, 0 ; m)=\{(\ldots, 0, m)\}$ and $Y_{-}\left(n_{1}, 0 ; m\right)=\emptyset$ if $m<n_{1}$ by Remark 6.3. Hence, for $1 \leq m \leq n_{1}$,

$$
\# Y_{-}\left(n_{1}, 0 ; m\right)= \begin{cases}1 & \text { if } m=n_{1} \\ 0 & \text { if } m<n_{1}\end{cases}
$$

Assume that $1 \leq m \leq n_{1}$ and $n_{2} \geq 1$. Let $y=\left(\ldots, y_{3}, y_{2}, y_{1}\right) \in Y_{-}\left(n_{1}, n_{2} ; m\right)$. Then, $y$ is of the form $y=\left(\ldots, y_{3}, y_{2}, m\right)$. By Remark 6.3 and the assumption that $n_{2} \geq 1$, we have $y_{2} \geq 1$. By the definition of $Y_{-}$, we obtain $y_{2} \leq F\left(y_{1}\right)=F(m)$. Moreover, we have $y_{2} \leq n_{2}$ since $y \in Y_{-}\left(n_{1}, n_{2} ; m\right)$. Hence, $1 \leq y_{2} \leq \min \left\{F(m), n_{2}\right\}$. Then we have

$Y_{-}\left(n_{1}, n_{2} ; m\right)=\bigsqcup_{l=1}^{\min \left\{F(m), n_{2}\right\}}\left\{\left(\ldots, y_{3}, l, m\right) \in Y_{-} \mid m+\sum_{j=3,5, \ldots} y_{j}=n_{1}, l+\sum_{j=4,6 \ldots} y_{j}=n_{2}\right\}$. 
For $1 \leq l \leq \min \left\{F(m), n_{2}\right\}$, we see that

$$
\begin{aligned}
& \#\left\{\left(\ldots, y_{4}, y_{3}, l, m\right) \in Y_{-} \mid m+\sum_{j=3,5, \ldots} y_{j}=n_{1}, l+\sum_{j=4,6 \ldots} y_{j}=n_{2}\right\} . \\
= & \#\left\{\left(\ldots, y_{4}, y_{3}, l\right) \in Y_{-} \mid \sum_{j=3,5, \ldots} y_{j}=n_{1}-m, l+\sum_{j=4,6 \ldots} y_{j}=n_{2}\right\} . \\
= & \# Y_{-}\left(n_{2}, n_{1}-m ; l\right) .
\end{aligned}
$$

Hence we obtain

$$
\# Y_{-}\left(n_{1}, n_{2} ; m\right)=\sum_{l=1}^{\min \left\{F(m), n_{2}\right\}} \# Y_{-}\left(n_{2}, n_{1}-m ; l\right)
$$

If $n_{1}-m=0$, then we see by (6.5) that $\# Y_{-}\left(n_{2}, n_{1}-m ; l\right)$ becomes a finite sum of 0 and 1. Assume that $n_{1}-m>0$. We set $n_{1}^{\prime}:=n_{2}$ and $n_{2}^{\prime}:=n_{1}-m$. Let $m^{\prime}$ be such that $1 \leq m^{\prime} \leq \min \left\{F(m), n_{2}\right\}$. Since $1 \leq m^{\prime} \leq n_{1}^{\prime}$ and $n_{2}^{\prime} \geq 1$, we obtain, by the same argument as above,

$$
\begin{aligned}
\# Y_{-}\left(n_{1}^{\prime}, n_{2}^{\prime} ; m^{\prime}\right) & =\sum_{l=1}^{\min \left\{F\left(m^{\prime}\right), n_{2}^{\prime}\right\}} \# Y_{-}\left(n_{2}^{\prime}, n_{1}^{\prime}-m^{\prime}, l\right) \\
& =\sum_{l=1}^{\min \left\{F\left(m^{\prime}\right), n_{1}-m\right\}} \# Y_{-}\left(n_{1}-m, n_{2}-m^{\prime}, l\right) .
\end{aligned}
$$

Because $n_{2}-m^{\prime} \leq n_{2}-1$, this process ends after at most $n_{2}$ steps. Similarly, we can compute $\# Y_{+}\left(n_{1}, n_{2} ; m\right)$.

\subsection{Number of elements in $\mathcal{B}(\lambda)_{\mu}$.}

We set

$$
\begin{aligned}
& Z(\lambda)_{-}:=\left\{y \in \mathbb{Z}_{\iota^{+}}^{+\infty} \mid y \text { is of the form either (国) or }(\underline{b})\right\}, \\
& \left.Z(\lambda)_{+}:=\left\{y \in \mathbb{Z}_{\iota^{-}}^{-\infty} \mid y \text { is of the form either (다 }\right) \text { or }(\underline{d})\right\} \text {, }
\end{aligned}
$$

where

(a) $\left(\ldots, 0, p_{m}, \ldots, p_{2}, p_{1}\right)$ for some $1 \leq m$;

(b) $\left(\ldots, 0, q_{m}, \ldots, q_{n+2}, q_{n+1}, p_{n}, \ldots, p_{2}, p_{1}\right)$ for some $n, m \in \mathbb{Z}$ such that $1 \leq n<m$, where $q_{m}, q_{m-1}, \ldots, q_{n+1}$ are integers satisfying that $0<q_{j}<p_{j}$ for $n+1 \leq j \leq m$, and $q_{j+1} / p_{j+1}<q_{j} / p_{j}$ for $n+1 \leq j \leq m-1$; 
(c) $\left(-p_{0},-p_{-1}, \ldots,-p_{m+1}, q_{m}-p_{m}, q_{m-1}-p_{m-1}, \ldots, q_{n+1}-p_{n+1}, 0, \ldots\right)$ for some $n, m \in \mathbb{Z}$ such that $n<m \leq-1$, where $q_{m}, q_{m-1}, \ldots, q_{n+1}$ are integers satisfying that $0<q_{j}<$ $p_{j}$ for $n+1 \leq j \leq m$, and $q_{j+1} / p_{j+1}<q_{j} / p_{j}$ for $n+1 \leq j \leq m-1$;

(d) $\left(-p_{0},-p_{-1}, \ldots,-p_{n+1}, 0, \ldots\right)$ for some $n \leq-1$.

Let

$$
\begin{aligned}
& Y(\lambda)_{-}:=\left\{\left(\ldots, 0, y_{m}, \ldots y_{2}, y_{1}\right) \in Y_{-} \mid y_{1}=1\right\} \\
& Y(\lambda)_{+}:=\left\{\left(y_{0}, y_{-1}, \ldots, y_{j}, \ldots,\right) \in Y_{+} \mid y_{0}=-1\right\} .
\end{aligned}
$$

Proposition 6.4. It hold that $Z(\lambda)_{-}=Y(\lambda)_{-}$and $Z(\lambda)_{+}=Y(\lambda)_{+}$.

Proof. We give a proof only for $Z(\lambda)_{-}=Y(\lambda)_{-}$; the proof for $Z(\lambda)_{+}=Y(\lambda)_{+}$is similar. By (6.2) and Lemma 6.1, we can easily check that $Z(\lambda)_{-} \subset Y(\lambda)_{-}$. We show the reverse inclusion $Z(\lambda)_{-} \supset Y(\lambda)_{-}$. Let $y \in Y(\lambda)_{-}$. By Remark 6.3 and the definition of $Y(\lambda)_{-}$, the element $y$ is of the form $y=\left(\ldots, 0, y_{m}, \ldots y_{2}, y_{1}\right)$ for some $m \in \mathbb{Z}_{\geq 1}$ with $y_{j}>0$ for all $1 \leq j \leq m$. By $y_{1}=1=p_{1}$ and (6.2), we see that $y_{2} \leq F\left(y_{1}\right) \leq F\left(p_{1}\right)=p_{2}$, where we use the monotonicity of $F$ (see Corollary 6.2). Similarly, we see by $y_{2} \leq p_{2}$ and (6.2) that $y_{3} \leq F\left(y_{2}\right) \leq F\left(p_{2}\right)=p_{3}$. Repeating this argument, we obtain

$$
1 \leq y_{j} \leq p_{j} \quad \text { for all } 1 \leq j \leq m
$$

Let $n$ be the largest integer $n^{\prime} \leq m$ such that $y_{n^{\prime}}=p_{n^{\prime}}$; note that $y_{1}=1=p_{1}$. Since $y \in Y_{-}$and (6.6) , we have $p_{n} \leq F\left(y_{n-1}\right) \leq F\left(p_{n-1}\right)=p_{n}$. Hence we obtain $F\left(y_{n-1}\right)=p_{n}$. Since $1=F\left(y_{n-1}\right) / p_{n} \leq y_{n-1} / p_{n-1}$ by Lemma 6.1 and this equality, we get $p_{n-1} \leq$ $y_{n-1}$. Hence, by (6.6), we have $p_{n-1}=y_{n-1}$. Repeating this argument, we obtain $p_{j}=$ $y_{j}$ for all $1 \leq j \leq n$. If $n=m$, then we obtain $y=\left(\ldots, 0, p_{m}, \ldots p_{2}, p_{1}\right)$, which is of the form (a) , and hence $y \in Z(\lambda)_{-}$. Assume that $n<m$. Then we have $y=$ $\left(\ldots, y_{m}, \ldots, y_{n+1}, p_{n}, \ldots p_{1}\right)$, where

$$
1 \leq y_{j}<p_{j} \text { for all } n+1 \leq j \leq m .
$$

Since $y \in Y_{-}$, we see by Lemma 6.1 that $y_{j+1} / p_{j+1} \leq y_{j} / p_{j}$ for all $n+1 \leq j \leq m-1$. Suppose, for a contradiction, that $y_{j+1} / p_{j+1}=y_{j} / p_{j}$. Then, $y_{j+1}=y_{j} p_{j+1} / p_{j}$. We see by $p_{0}=p_{1}=1$ and (4.1) that $p_{j}$ and $p_{j+1}$ are relatively prime (see [1, Lemma $\left.4.5(1)\right]$ ). Because $y_{j+1}$ is a positive integer, we obtain $y_{j} \geq p_{j}$, which contradicts (6.7). Therefore, we obtain $y_{j+1} / p_{j+1}<y_{j} / p_{j}$ for all $n+1 \leq j \leq m-1$. Thus we see that $y$ is of the form (b), and hence $y \in Z(\lambda)_{-}$. Thus we have proved the proposition.

Let $\mu=\lambda-n_{1} \alpha_{1}-n_{2} \alpha_{2}$ with $n_{1}, n_{2} \in \mathbb{Z}_{\geq 0}$, and assume that $\mu \neq \lambda$. By the results of Section 5 and the fact that $p_{1}=1$ (and hence there is no integer $q$ such that $0<q<p_{1}$ ), 
there exists a natural bijection from $\mathcal{B}(\lambda)_{\mu}$ onto

$$
\left\{\left(\ldots, 0, y_{m}, \ldots y_{2}, y_{1}\right) \in Z(\lambda)_{-} \mid \sum_{j: \text { odd }} y_{j}=n_{1}, \sum_{j: \text { even }} y_{j}=n_{2}\right\} .
$$

Moreover, we see that

$$
\begin{aligned}
Y_{-}\left(n_{1}, n_{2} ; 1\right) & =\left\{\left(\ldots, 0, y_{m}, \ldots y_{2}, y_{1}\right) \in Y_{-} \mid y_{1}=1, \sum_{j: \text { odd }} y_{j}=n_{1}, \sum_{j: \text { even }} y_{j}=n_{2}\right\} \\
& =\left\{\left(\ldots, 0, y_{m}, \ldots y_{2}, y_{1}\right) \in Y(\lambda)_{-} \mid \sum_{j: \text { odd }} y_{j}=n_{1}, \sum_{j: \text { even }} y_{j}=n_{2}\right\} .
\end{aligned}
$$

By Proposition 6.4, we see that $\# \mathcal{B}(\lambda)_{\mu}=\# Y_{-}\left(n_{1}, n_{2} ; 1\right)$. Similarly, if $\mu=\lambda-n_{1} \alpha_{1}-n_{2} \alpha_{2}$ with $n_{1}, n_{2} \in \mathbb{Z}_{\leq 0}$, and $\mu \neq \lambda$, then $\# \mathcal{B}(\lambda)_{\mu}=\# Y_{+}\left(n_{1}, n_{2} ;-1\right)$. Summarizing these, we obtain the following theorem.

Theorem 6.5. For $\mu \in P$, it holds that

$$
\# \mathcal{B}(\lambda)_{\mu}= \begin{cases}1 & \text { if } \mu=\lambda \\ \# Y_{-}\left(n_{1}, n_{2} ; 1\right) & \text { if } \mu \neq \lambda \text { and } \mu=\lambda-n_{1} \alpha_{1}-n_{2} \alpha_{2} \text { for some } n_{1}, n_{2} \geq 0 \\ \# Y_{+}\left(n_{1}, n_{2} ;-1\right) & \text { if } \mu \neq \lambda \text { and } \mu=\lambda-n_{1} \alpha_{1}-n_{2} \alpha_{2} \text { for some } n_{1}, n_{2} \leq 0 \\ 0 & \text { otherwise. }\end{cases}
$$

\section{Acknowledgment.}

The author would like to thank Daisuke Sagaki, who is his supervisor, for his kind support and advice.

\section{References}

[1] R. Hiasa, Connectedness of Lakshmibai-Seshadri path crystals for hyperbolic KacMoody algebras of rank 2, Comm. Algebra, 49, 2021, pp.772-789.

[2] J. Hong and S.-J. Kang, Introduction to quantum groups and crystal bases, Graduate Studies in Mathematics, 42, Amer. Math. Soc., 2002.

[3] M. Ishii, S. Naito, and D. Sagaki, Semi-infinite Lakshmibai-Seshadri path model for level-zero extremal weight modules over quantum affine algebras, Adv. Math., 2016, pp.967-1009.

[4] A. Joseph, Quantum groups and their primitive ideals, Ergebnisse der Mathematik und ihrer Grenzgebiete (3), 29, Springer-Verlag, 1995. 
[5] M. Kashiwara, Crystal bases of modified quantized enveloping algebra, Duke Math. J., 73, 1994, pp.383-413.

[6] M. Kashiwara, The crystal base and Littelmann's refined Demazure character formula, Duke Math. J., 71, 1993, pp.839-858.

[7] M. Kashiwara, On crystal bases, in "Representation of groups", CMS Conf. Proc., 16, pp.155-197, Amer. Math. Soc., Providence, RI, 1995.

[8] M. Kashiwara, Similarity of crystal bases, in "Lie algebras and their representation", Contemp. Math., 194, pp.177-186, Amer. Math. Soc., Providence, RI, 1996.

[9] S. Naito and D. Sagaki, Path model for a level-zero extremal weight module over a quantum affine algebra, Int. Math. Res. Not., 2003, pp.1731-1754.

[10] S. Naito and D. Sagaki, Path model for a level-zero extremal weight module over a quantum affine algebra II, Adv. Math., 200, 2006, pp.102-124.

[11] T. Nakashima and A. Zelevinsky, Polyhedral realizations of crystal bases for quantized Kac-Moody algebras, Adv. Math., 131, 1997, pp.253-278.

[12] P. Littelmann, A Littlewood-Richardson rule for symmetrizable Kac-Moody algebras, Invent. Math., 116, 1994, pp.329-346.

[13] P. Littelmann, Paths and root operators in representation theory, Ann. of Math., (2), 142, 1995, pp.499-525.

[14] D. Sagaki and D. Yu, Path model for an extremal weight module over the quantized hyperbolic Kac-Moody algebra of rank 2, Comm. Algebra, 49, 2021, pp.690-705.

[15] D. Yu, Lakshmibai-Seshadri paths for hyperbolic Kac-Moody algebras of rank 2, Comm. Algebra, 46, 2018, pp.2702-2713. 